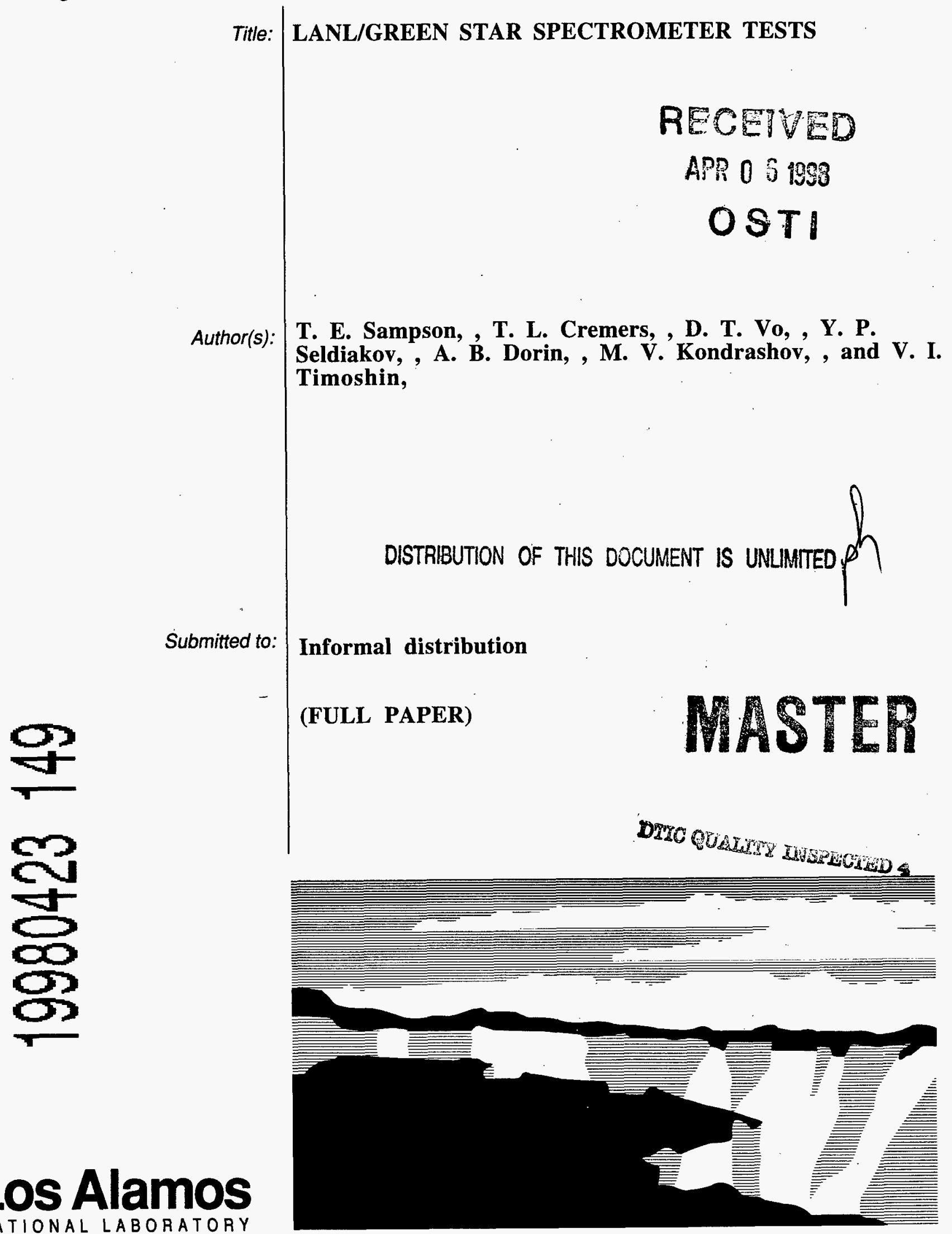




\section{DISCLAIMER}

This report was prepared as an account of work sponsored by an agency of the United States Government. Neither the United States Government nor any agency thereof, nor any of their employees, makes any warranty, express or implied, or assumes any legal liability or responsibility for the accuracy, completeness, or usefulness of any information, apparatus, product, or process disclosed, or represents that its use would not infringe privately owned rights. Reference herein to any specific commercial product, process, or service by trade name, trademark, manufacturer, or otherwise does not necessarily constitute or imply its endorsement, recommendation, or favoring by the United States Government or any agency thereof. The views and opinions of authors expressed herein do not necessarily state or reflect those of the United States Government or any agency thereof. 


\title{
LANL/GREEN STAR SPECTROMETER TESTS
}

by

\author{
T. E. Sampson, T. L. Cremers, Duc T. Vo \\ Los Alamos National Laboratory \\ Los Alamos, New Mexico, USA \\ Y. P. Seldiakov, A. B. Dorin, M. V. Kondrashov \\ Green Star \\ Moscow, Russia \\ V. I. Timoshin \\ VNIINM \\ Moscow, Russia
}

\section{Introduction}

The United States and Russia have agreed to the joint development of a nondestructive assay system for use to support the dismantlement of nuclear weapons in Russia. This nondestructive assay system will be used to measure plutonium produced by the conversion of Russian nuclear weapons. The NDA system for Russia will be patterned after the ARIES NDA system being constructed at Los Alamos. One goal of the program is to produce an NDA system for use in Russia that maximizes the use of Russian resources to facilitate maintenance and future upgrades.

The Green Star SBS50 Single Board Spectrometer system (Green Star Ltd., Moscow, Russia) has been suggested for use as the data acquisition component for gamma ray instruments in the system. Possible uses are for plutonium isotopic analysis and also segmented gamma scanning.

Green Star has also developed analysis software for the SBS50. This software, both plutonium isotopic analysis and uranium enrichment analysis, was developed specifically for customs/border inspection applications (low counting rate applications and identification as opposed to quantification) and was not intended for MC\&A applications. Because of the relative immaturity of the Green Star plutonium isotopic analysis software (it has been under development for only one year and is patterened after US development circa 1980), it was tentatively agreed, before the tests, that the Russian NDA system would use the Los Alamos PC/FRAM software for plutonium isotopic analysis. However, it was also decided to include the Green Star plutonium isotopic software in the testing, both to quantify its performance for MC\&A applications and also to provide additional data to Green Star for further development of their software. The main purpose of the testing was to evaluate the SBS-50 spectrometer as a data acquisition device for use with LANL software.

The purpose of the tests reported here are:

1. Test performance of Green Star spectrometer with respect to resolution and throughput as a function of counting rate using both planar and coaxial detectors.

2. Compare the Green Star plutonium isotopic analysis software with the LANL PC/FRAM software.

3. Test the Green Star spectrometer as a front end data acquisition device for use with the LANL PC/FRAM software.

4. Based on the results of the tests, make a recommendation for the gamma spectroscopy hardware and software to be used in the Russian NDA system.

Los Alamos Data Acquisition System Descriptions

Three Los Alamos detector/data acquisition systems were provided.

Los Alamos planar HPGe detector system

Canberra SN 11892067, $16 \mathrm{~mm}$ diameter by $13 \mathrm{~mm}$ deep planar detector, resolution $500 \mathrm{eV}$ at $6 \mathrm{us,} 1000 \mathrm{cps}$. 
ORTEC 4001C/4002D NIM Bin and power supply

ORTEC 459 High Voltage Bias supply

ORTEC 672 Spectroscopy amplifier

Canberra 8077450 MHZ ADC

Canberra 8232 Digital Stabilizer

Canberra 2071A Counter/Timer

Canberra S100 MCA

IBM 760L Think Pad computer with Dock 1 Docking Station

Hewlett/Packard Laser Jet $5 \mathrm{~L}$ printer

PC/FRAM version 2.2 isotopic analysis software

Los Alamos coaxial HPGe Hand Held detector system "Coaxial System 1"

Canberra SN 5963603, $57 \mathrm{~mm}$ dia by $40.5 \mathrm{~mm}$ long coaxial detector, resolution $733 \mathrm{eV}$ at 6 us, $1000 \mathrm{cps}$ for ${ }^{57} \mathrm{Co}, 1.74 \mathrm{keV}$ at $6 \mathrm{us}, 1000 \mathrm{cps},{ }^{60} \mathrm{Co}$.

BNC AP3 PortaNIM Bin and power supply

ORTEC 459 High Voltage Bias supply

ORTEC 672 Spectroscopy amplifier

Canberra $8077450 \mathrm{MHZ}$ ADC

Canberra 8232 Digital Stabilizer

Canberra 2071A Counter/Timer

Canberra S100 MCA

Computer: IBM 755CE Think Pad with Dock 1 Docking Station

Hewlett/Packard Laser Jet 5L printer

PC/FRAM version 2.1 isotopic analysis software

Los Alamos second coaxial HPGe detector system "Coaxial System 2"

Detector: $60 \mathrm{~mm}$ dia by $42 \mathrm{~mm}$ long coaxial, Canberra SN 9953619 , resolution $718 \mathrm{eV}$ at 6 us, $1000 \mathrm{cps}$ for ${ }^{57} \mathrm{Co}$, $1.75 \mathrm{keV}$ at $6 \mathrm{us}, 1000 \mathrm{cps},{ }^{60} \mathrm{Co}$.

ORTEC DSPEC Digital Gamma Ray Spectrometer

Computer: IBM 760XD Think Pad ${ }^{-}$

Hewlett/Packard Laser Jet 4 Plus

PC/FRAM version 2.2 isotopic analysis software

\section{Green Star Data Acquisition System Description}

Green Star brought three SBS-50 systems installed in notebook computers and an extra SBS-50 board.

The single board spectrometer SBS-50 is a standard ISA board with the length $136 \mathrm{~mm}$ (weight $200 \mathrm{~g}$ ). The board can be installed in most IBM-compatible desktop computers and suitable IBM-compatible notebook computers. The SBS-50 board contains: a P/Z network, a spectroscopy amplifier with time-variant filters, a gated base-line restorer, a $100 \mathrm{MHz}$ Wilkinson $\mathrm{ADC}$, a high voltage power supply for the detector, a low voltage power supply for the preamplifier, computer interface circuits. An optional pile-up rejector is available but was not included in the systems tested.

The main performance characteristics are given in the following table (typical performance values are in parentheses). 


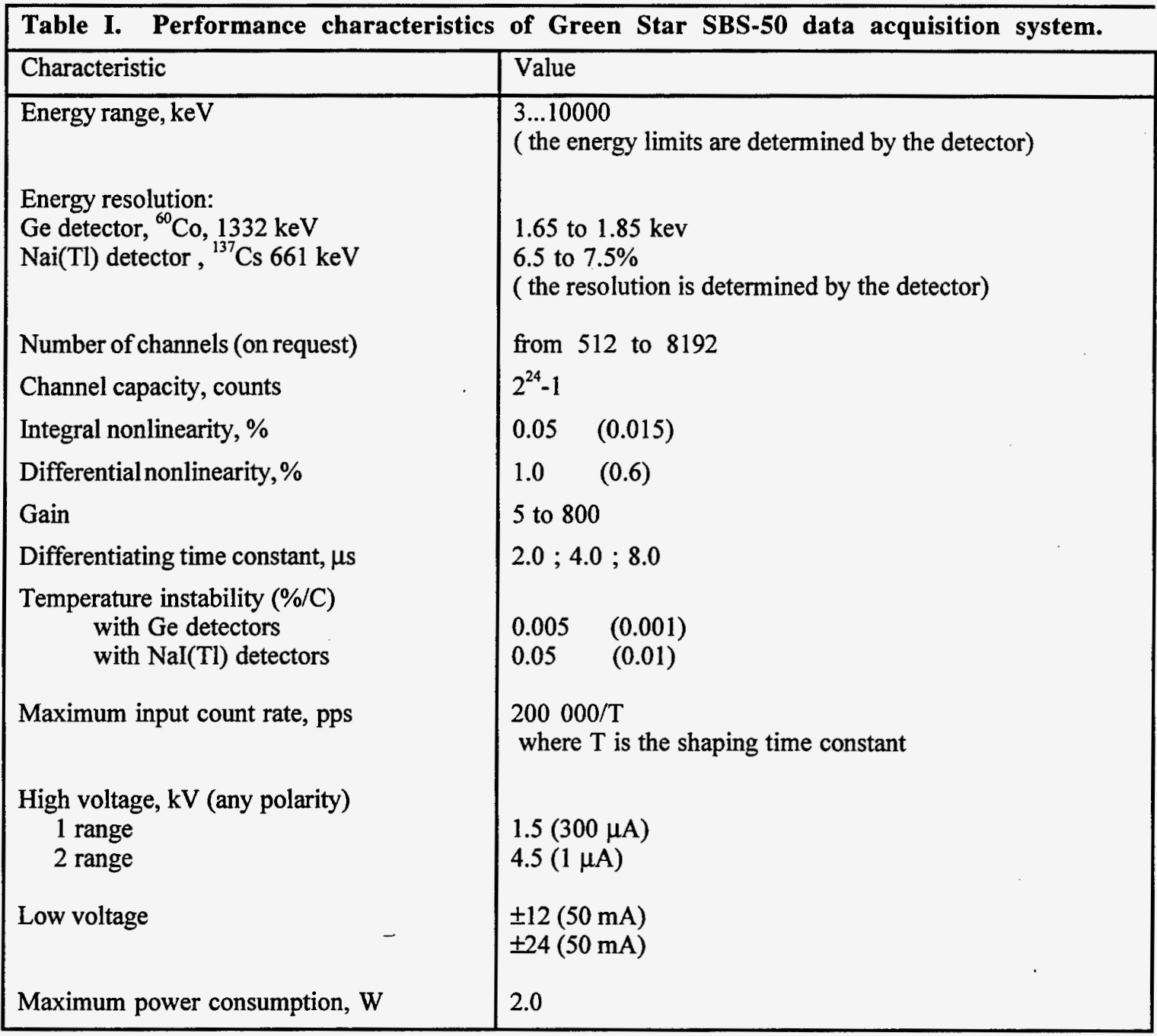

All spectrometers were supplied with necessary software developed by Green Star for qualitative and quantitative spectra analysis, complete gamma radionuclide analysis and applied programs for plutonium isotope composition analysis and uranium enrichment determination.

Three SBS-50 boards were installed in notebook computers model CD-6000; manufactured by MD\&I Corporation 9582, Telstar Ave., El Monte CA, 91731, USA. The fourth SBS-50 system was operated with a LANL desktop computer, Compaq Deskpro 66M.

\section{Data Collection System Interconnections}

The systems were set up to present the same data stream to both the LANL and Green Star data acquisition systems. High voltage and low voltage power was supplied to the detector by the Green Star system. The "Energy" output from the preamp (93 $\Omega$ ) was fed to the Green Star system while the "Timing" output from the preamp (50 $\Omega$ ) was fed to the amplifier input of the LANL electronics. A counter/timer in the Los Alamos NIM bin systems (planar detector and coaxial detector 1) monitored the count rate at the preamplifier output. A separate mini NIM bin with ORTEC 672 amplifier and Canberra 2071A counter/timer was used with coaxial detector 2 in parallel with the DSPEC electronics to monitor the count rate because the ORTEC DSPEC data acquisition system does not display the counting rate. 
These interconnections are shown in Fig. 1.

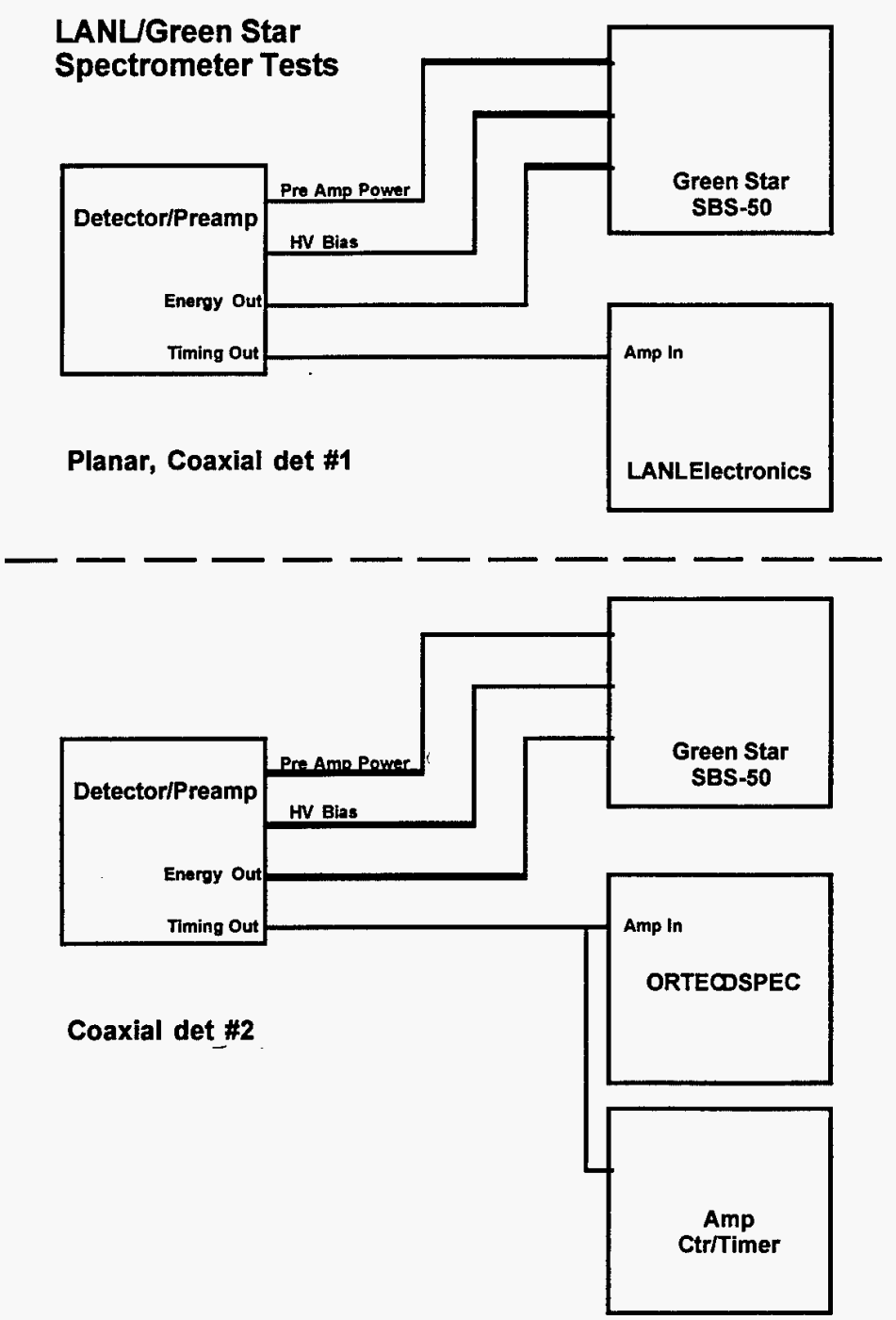

Fig. 1. Electronics Interconnections for LANL/Green Star Spectrometer Tests. 


\section{Sample Characteristics}

The isotopic fractions, americium concentration, $\mathrm{P}_{\text {eff }}$ and ${ }^{240} \mathrm{Pu}_{\text {eff }}$ of the standards used in this study are listed below. The samples were selected based on availability and the desire to make the majority of measurements on low burnup samples expected to be characteristic of plutonium from dismantled weapons. Some uranium samples were also included because both the Green Star and Los Alamos software have uranium measurement capability.

Samples with complete and well-documented isotopic distribution analyses are scarce in Russia. Below we describe the source of the values listed below in Table II. Uranium standards are also described even though we do not report uranium results in this document. Green Star took the opportunity to measure these well-documented standards to help enhance their uranium isotopic analysis software. LANL and Green Star uranium measurements were not comparable and are not reported here.

Table II. Isotopic Analysis and Other Information for LANL/Green Star tests as of October 8, 1997

\begin{tabular}{|c|c|c|c|c|c|c|c|c|}
\hline & \multicolumn{8}{|c|}{ Plutonium Samples 08-Oct-1997 } \\
\hline & SGA30 & PUEU7 & METSPH & STD118 & CBNM93 & CBNM84 & CBNM70 & CBNM61 \\
\hline$w t \%$ Pu-238 & 0.0059 & 0.0135 & $0.0133^{*}$ & 0.0243 & 0.0107 & 00646 & 079271 & 11281 \\
\hline wt $\% \mathrm{Pu}-239$ & 96.3504 & 93.8749 & 93.8479 & 90.5520 & 93.5061 & 84.7192 & 75.1104 & 64.4139 \\
\hline$w t \% \mathrm{Pu}-240$ & 3.5609 & 5.8638 & 5.9529 & 9.0101 & 6.3140 & 14.2588 & 18.7252 & 26.1504 \\
\hline $\mathrm{wt} \% \mathrm{Pu}-241$ & 0.0645 & 0.1818 & 0.1524 & 0.3090 & 0.1296 & 0.5981 & 3.2431 & 3.9872 \\
\hline$w t \% \mathrm{Pu}-242$ & 0.0183 & 0.0659 & 0.0335 & 0.1046 & 0.0396 & 0.3593 & 2.1286 & 4.3204 \\
\hline$w t \% \mathrm{Pu}-244$ & 0 & 0 & 0 & 0 & 0 & 0 & 0 & 0 \\
\hline ppm Am-241/Pu & 630.9 & 1170.3 & 3266.4 & 4450.3 & 1962.0 & 6445.9 & 35104.7 & 43303 \\
\hline Approximate grams $\mathrm{Pu}$ & 30.00 & 9.99 & 9.25 & 1.59 & 5.99 & 5.97 & 6 & 6 \\
\hline $\begin{array}{l}\text { EffectiveSpecificPower } \\
(\mathrm{mW} / \mathrm{gPu})\end{array}$ & 2.21844 & 2.44224 & 2.68542 & 3.04143 & 2.54007 & 3.76756 & 11.3962 & 14.58345 \\
\hline $\begin{array}{l}\text { Fraction[Pu-240 } \\
\text { (effective)] }\end{array}$ & 0.036065 & 0.060085 & 0.060427 & 0.092470 & 0.064074 & 0.150253 & 0.242989 & 0.362515 \\
\hline
\end{tabular}

* Mass spec value from 1974. Suspected to be high.

\begin{tabular}{|c|c|c|c|c|c|}
\hline & \multicolumn{4}{|c|}{ Uranium Samples } & \\
\hline & CBNM031 & NBL-5 & UISO-52 & NBL-6 & NBL-7 \\
\hline$w t \% U-234$ & 0.0020 & 0.15 & 0.5306 & 0.370 & 0.979 \\
\hline$w t \% U-235$ & 0.3166 & 20.06 & 52.1174 & 52.560 & 93.183 \\
\hline$w t \% U-236$ & 0.0146 & 0.1900 & 0.8676 & 0.270 & 0.2960 \\
\hline$w t \% U-238$ & 99.6668 & 79.600 & 46.4844 & 46.800 & 5.5420 \\
\hline Approximate grams $U$ & 200 & 230.0 & 989 & 230 & 230.0 \\
\hline
\end{tabular}


SGA30 This sample, a mixture of $\mathrm{PuO}_{2}$ and diatomaceous earth, is one of a set used primarily for the calibration of Segmented Gamma-Scanners (SGS). The isotopic composition of the plutonium in this sample was determined independently by three US laboratories in 1990.

PUEU7 This sample is one of a set of mixed plutonium/enriched uranium standards prepared in 1988. This sample is pure $\mathrm{PuO}_{2}$ and does not contain any uranium. All the reported isotopic values for plutonium were determined by mass spectrometry at Los Alamos using national standards (NBS-948) for calibration. Pu-238 values were also determined by radiochemistry and found to be identical to the reported mass spectrometry values. Am was analyzed by Isotope Dilution Mass Spectrometry (IDMS).

METSPH These samples were a set $(5 \mathrm{~g}, 2 \mathrm{~g}, 1 \mathrm{~g}, 0.5 \mathrm{~g}, 0.2 \mathrm{~g})$ of plutonium metal spheres fabricated about 1975 for use in testing pedestrian portal monitors. The single mass spectrometer isotopic composition measurement is thought to be biased high for ${ }^{238} \mathrm{Pu}$.

STD118 This standard was fabricated in 1979 from plutonium oxide. The single routine Los Alamos analysis has always been found to be consistent with other standards.

CBNM 93, 84, 70,61 These samples are internationally certified reference materials from the Institute of Reference Materials and Measurements (formerly Central Bureau of Nuclear Measurements) in Belgium. These samples are the most widely accepted standards for nondestructive plutonium isotopic distribution measurements that are available.

CBNM031 Internationally certified standard for uranium enrichment measurements.

NBL 5, 6.7 Uranium enrichment standards certified at New Brunswick Laboratory (the standards certification authority for the Department of Energy). The listed values are preliminary, do not represent the final certification, and may change after final certification.

UISO52 A Los Alamos standard with certified isotopic composition measurements from two laboratories.

\section{Resolution and Throughput Measurements}

The first measurements made were resolution and throughput measurements on all three detectors using ${ }^{57} \mathrm{Co}$ and ${ }^{60} \mathrm{Co}$ sources. These measurements were meant to be made for comparable time constants on both the LANL and Green Star systems. The time constants were chosen to be applicable in real measurement situations expected to be encountered in the Material Disposition Program.

Two microseconds with triangular shaping was used to obtain spectral data with all LANL NIM equipment. The Green Star system used quasi-trapezoidal shaping (gated integrator) equivalent to 1.7 us for the planar detector and 2.0 us for the coaxial detectors. Co-57 and ${ }^{60} \mathrm{Co}$ sources were presented to the detector and the full width at half maximum and the net peak areas at 122 and $1332 \mathrm{keV}$ were determined as a function of the input counting rate to the main amplifier.

The results of the 5-Oct-1997 tests are presented in the following tables and figures. Shaping times are actually 1.7 (planar) and 2.0 (coaxial) us for the Green Star systems. 


\begin{tabular}{|c|c|c|c|c|}
\hline \multicolumn{5}{|c|}{ Table III. Planar Detector, 2us, ${ }^{57} \mathbf{C o}$} \\
\hline & \multicolumn{2}{|c|}{ Resolution (eV) } & \multicolumn{1}{c|}{$122 \mathrm{keV}$ Net Peak Rate s $^{-1}$ ) } \\
\hline Input cps & LANL & Green Star & LANL & Green Star \\
\hline 1000 & 518 & 523 & 467 & 450 \\
\hline 10000 & 523 & 525 & 4379 & 3353 \\
\hline 30000 & 532 & 534 & 9353 & 4986 \\
\hline 50000 & 535 & 554 & 11049 & 4542 \\
\hline
\end{tabular}

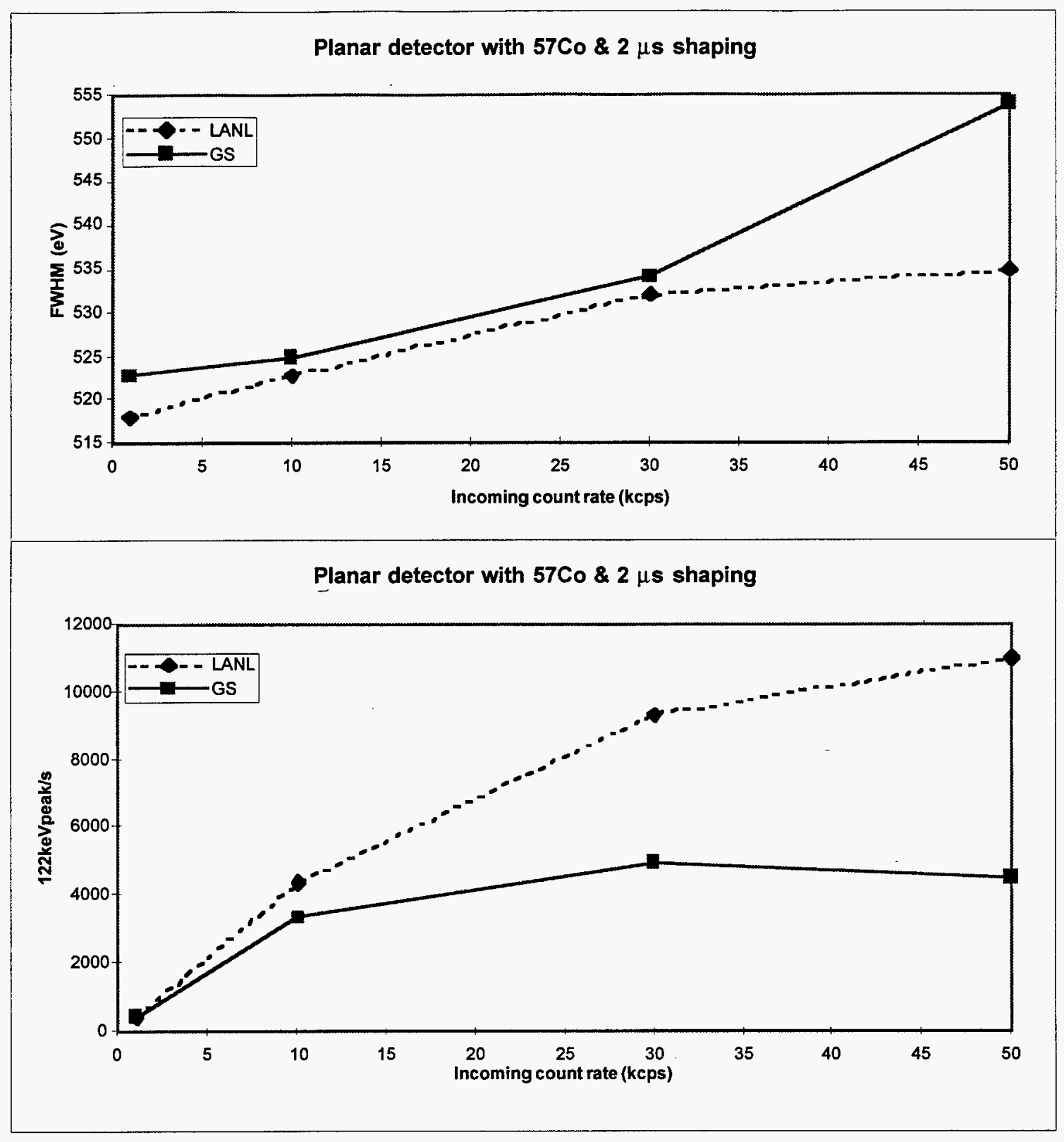

Fig. 2. Resolution and Throughput for Planar Detector, ${ }^{57} \mathrm{Co}$, 2us Shaping. 
Table IV. Coaxial Detector \#1, 2us shaping, ${ }^{60} \mathrm{Co}$

\begin{tabular}{|c|c|c|c|c|}
\hline & \multicolumn{2}{|c|}{ Resolution (keV) } & \multicolumn{2}{c|}{$1332 \mathrm{keV}$ Net Peak Rate $\left(\mathrm{s}^{-1}\right)$} \\
\hline Input cps & LANL & Green Star & LANL & Green Star \\
\hline 1000 & 1.97 & 1.84 & 54 & 52 \\
\hline 10000 & 1.98 & 1.89 & 552 & 375 \\
\hline 30000 & 2.01 & 2.08 & 1137 & 502 \\
\hline 50000 & 2.10 & 2.41 & 1271 & 353 \\
\hline
\end{tabular}

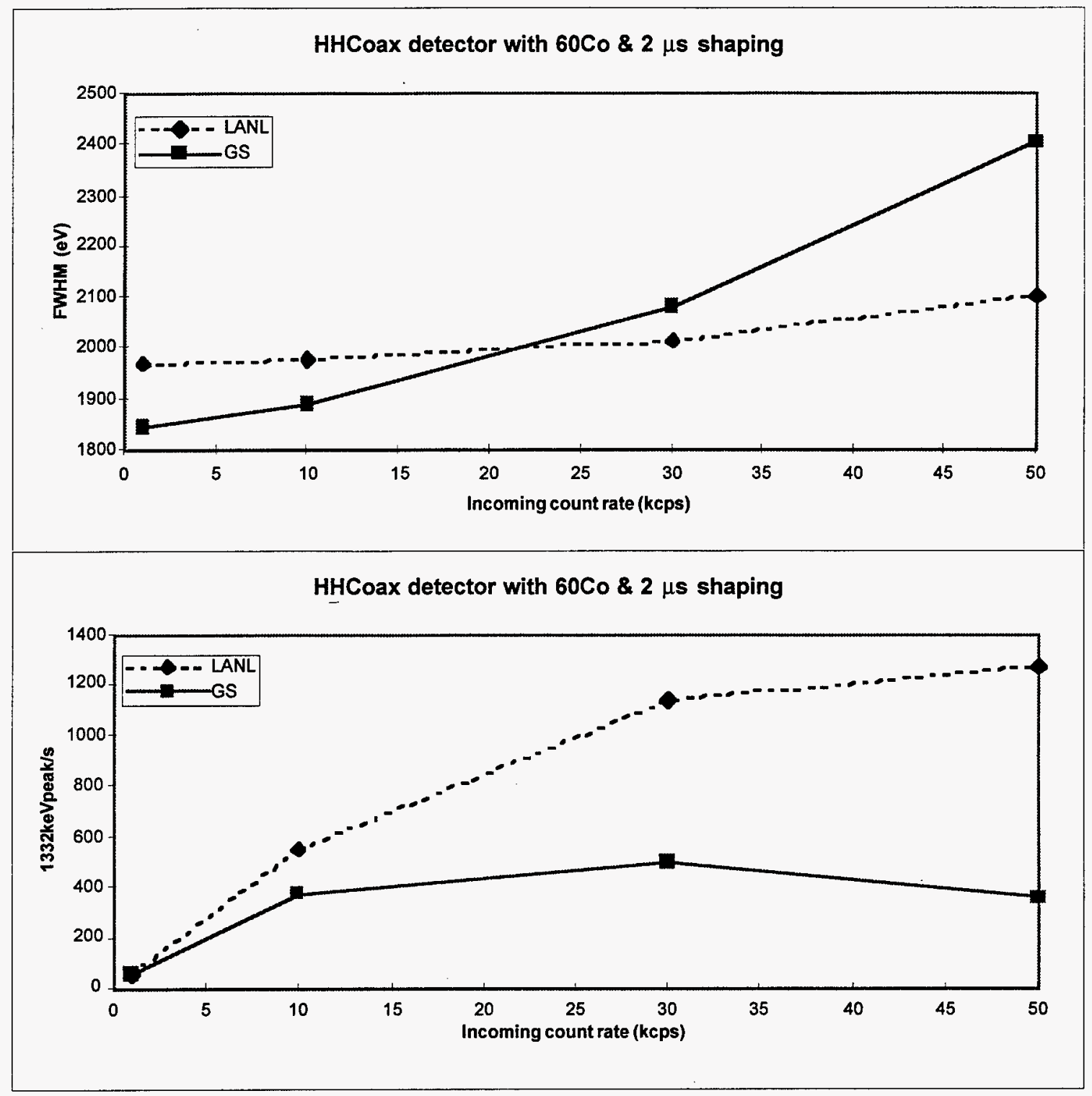

Fig. 3. Resolution and throughput for Coaxial Detector \#1 for ${ }^{60} \mathrm{Co}$. 
Table V. Coaxial Detector \#2, 2us shaping, ${ }^{57} \mathrm{Co}$

\begin{tabular}{|c|c|c|c|c|}
\hline & \multicolumn{2}{|c|}{ Resolution (eV) } & \multicolumn{2}{c|}{$122 \mathrm{keV}$ Net Peak Rate $\left(\mathrm{s}^{-1}\right)$} \\
\hline Input cps & LANL & Green Star & LANL & Green Star \\
\hline 1000 & 860 & 826 & 549 & 529 \\
\hline 10000 & 870 & 835 & 5337 & 3886 \\
\hline 30000 & 879 & 861 & 12011 & 5659 \\
\hline 50000 & 887 & 869 & 14059 & 4789 \\
\hline
\end{tabular}

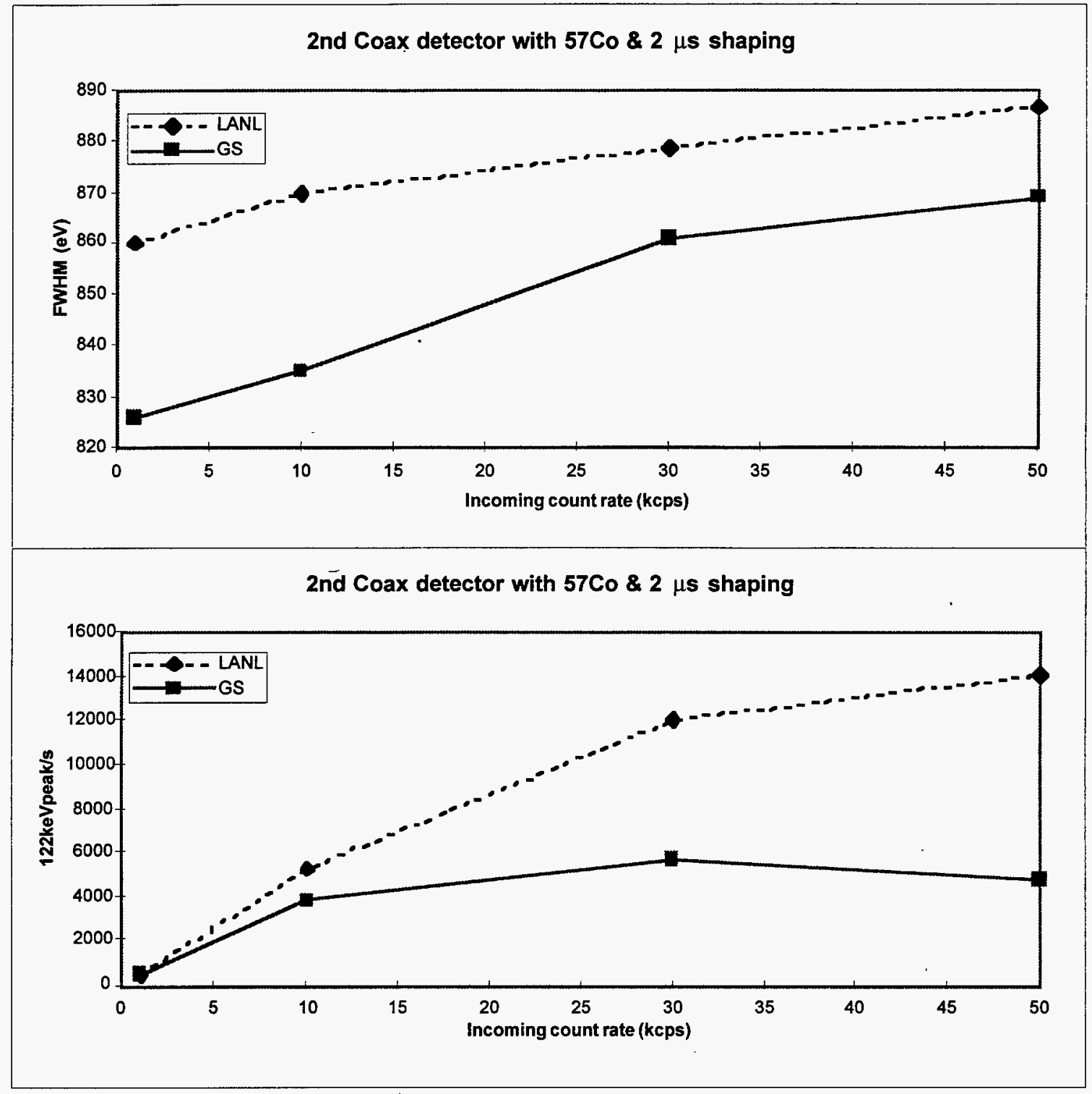

Fig. 4. Resolution and throughput for Coaxial Detector $\# 2$ for ${ }^{57} \mathrm{Co}$. 


\begin{tabular}{|c|c|c|c|c|}
\hline Table VI. Coaxial Detector \#2, 2us shaping, ${ }^{60} \mathrm{Co}$ \\
\hline & \multicolumn{2}{|c|}{ Resolution (keV) } & \multicolumn{1}{c|}{$1332 \mathrm{keV}$ Net Peak Rate $\left(\mathrm{s}^{-1}\right)$} \\
\hline Input cps & LANL & Green Star & LANL & Green Star \\
\hline 1000 & 1.83 & 1.81 & 62 & 59 \\
\hline 10000 & 1.85 & 1.89 & 640 & 398 \\
\hline 30000 & 1.85 & 1.94 & 1419 & 474 \\
\hline 50000 & 1.86 & 2.02 & 1647 & 347 \\
\hline
\end{tabular}

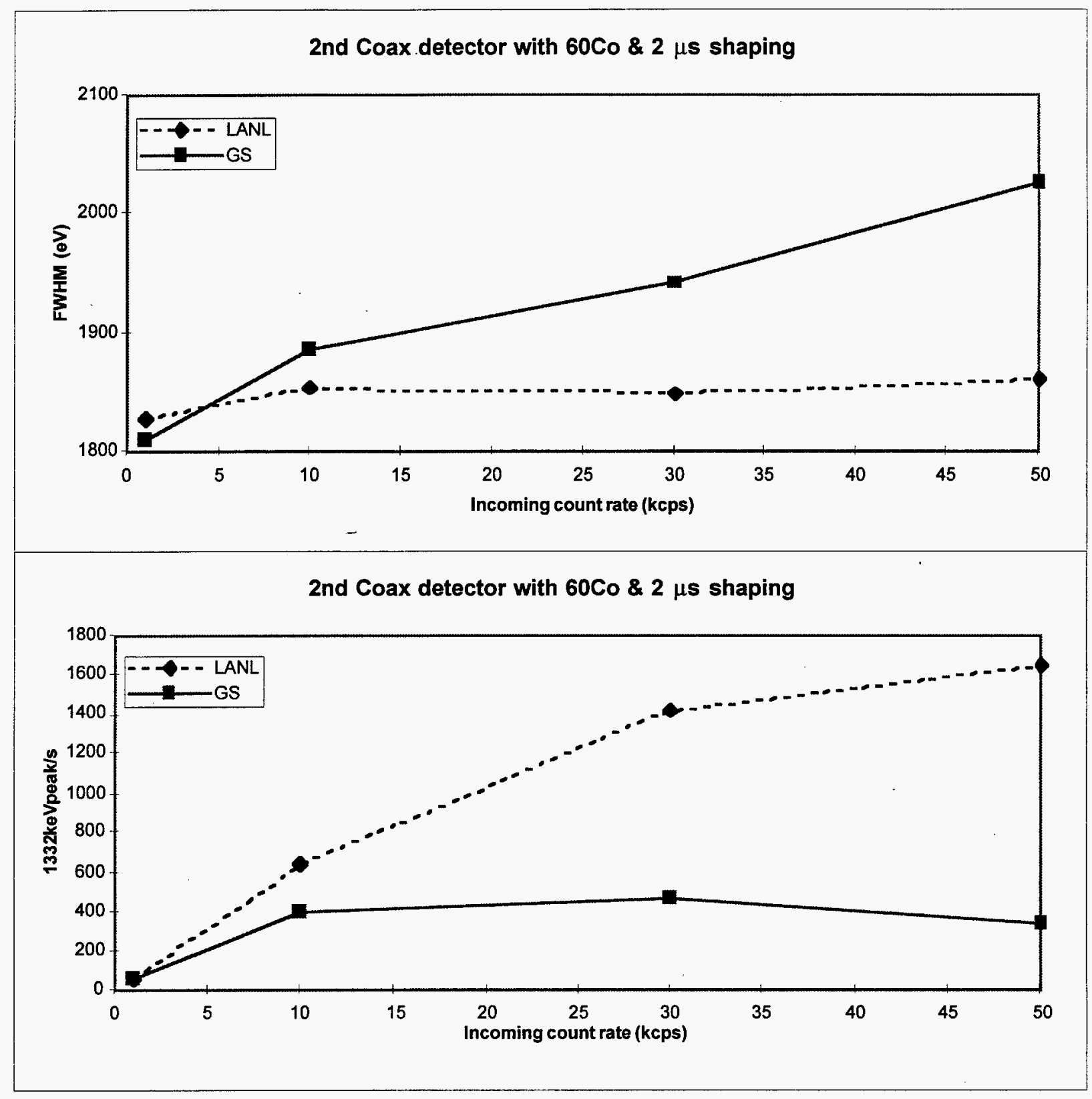

Fig. 5. Resolution and throughput for Coaxial detector $\# 2$ with ${ }^{60} \mathrm{Co}$. 


\section{Discussion of Resolution and Throughput Measurements}

One readily apparent difference between the Green Star systems and the LANL data acquisition systems was in the significantly lower throughput shown by the Green Star systems. One source of the lower throughput is the 100 $\mathrm{MHz}$ speed of the Green Star ADC compared the the $450 \mathrm{MHz}$ speed of two of the LANL systems and the optimized resolution/throughput of the third LANL system using a digital spectrometer. The throughput differences at $10 \mathrm{kHz}$ (the desired count rate for Green Star yielding 30\% dead time on their system) were not too significantally different between the two systems. The throughput differences at $-30 \mathrm{kHz}$ (the desired count rate for Los Alamos systems) were greater and led to noticeable differences in the measurement precision for acquisitions on the same sample at this higher counting rate.

In most cases the resolution of the Green Star systems was better at low count rates than the LANL electronics. This likely results from the different pulse shaping used in the Green Star amplifier compared to the LANL electronics.

The resolution and throughput curves shown above (Tables III-VI) represent the state of the systems for the data presented later. However, before the measurements started and after the measurements were completed Green Star made adjustments on the system used with Coaxial detector 1 as they felt the system was not optimally adjusted. Testing after each adjustment produced successively improved results as shown in Table VII.

\begin{tabular}{|c|c|c|c|c|c|c|}
\hline & \multicolumn{3}{|c|}{ Resolution $(\mathrm{keV})$} & \multicolumn{3}{|c|}{$1332 \mathrm{keV}$ Net Peak Rate $\left(\mathrm{s}^{-1}\right)$} \\
\hline Input cps & 1st Meas. & 2nd Meas. & 3rd Meas. & 1st Meas. & 2nd Meas. & 3rd Meas. \\
\hline 1000 & 1.86 & 1.84 & 1.77 & 51 & 52 & 52 \\
\hline 10000 & 1.99 & 1.89 & 1.83 & 379 & 375 & 384 \\
\hline 30000 & 2.26 & 2.08 & 1.92 & 484 & 502 & 477 \\
\hline 50000 & 2.54 & 2.41 & 2.03 & 376 & 353 & 366 \\
\hline
\end{tabular}




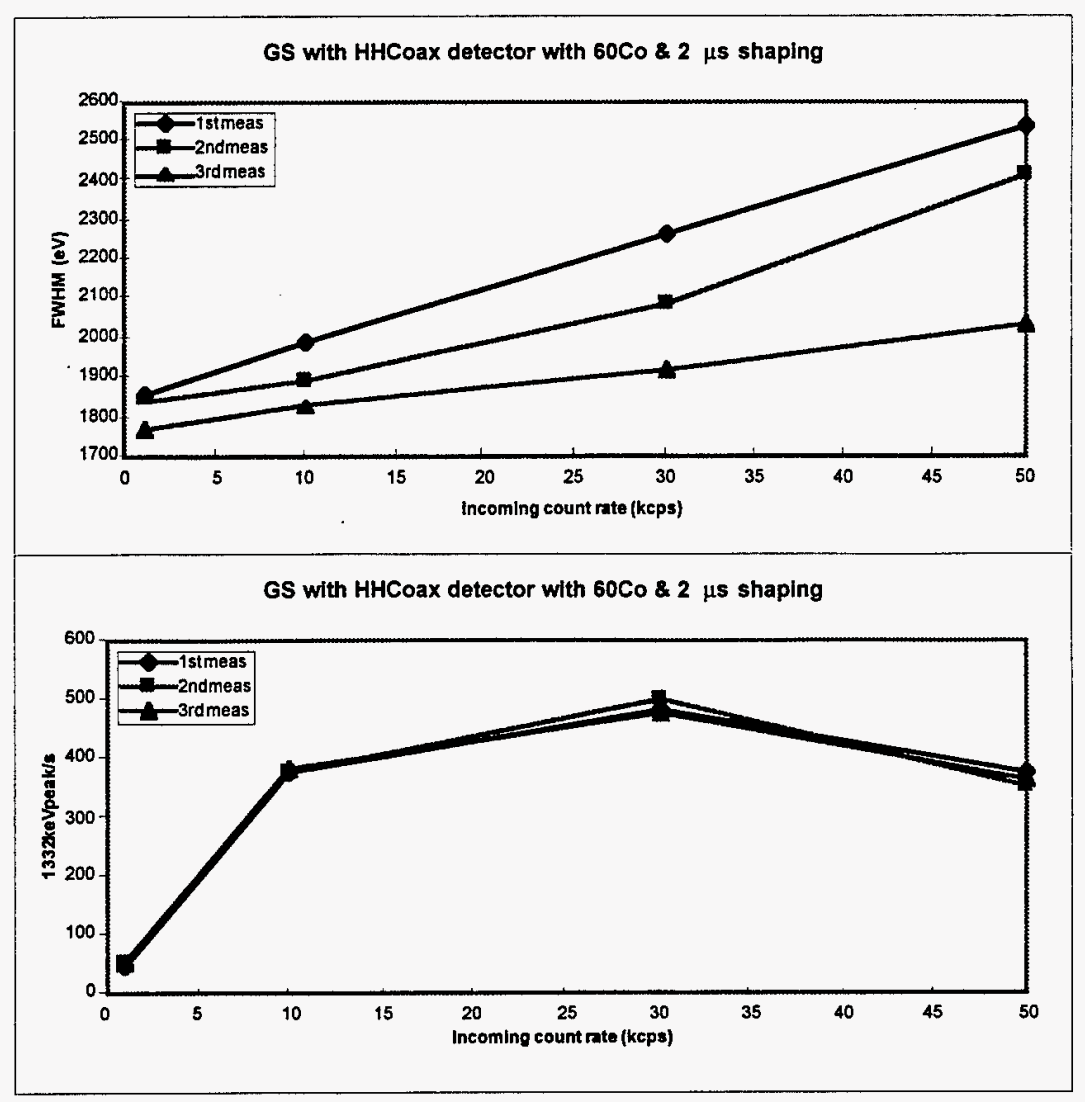

Fig. 6. Resolution and throughput for Coaxial Detector \#1 for three different adjustments of Green Star electronics.

The results in Table VII for 2nd Meas. are the same as in Table IV and represent the resolution during the measurements. Note that these adjustments left the throughput unaffected.

\section{Sample Measurement Protocol}

All measurements, both LANL and Green Star, were made for repeated one_hour counting times. The repeated measurements were made automatically without sample replacement. The standard deviations quoted are the standard deviation for a single measurement as determined from the statistical spread of the repeated runs. They are NOT the standard deviations reported by the software. The number of repeated measurements per sample varied from 3 to 21 with the majority being from 10-15 repeats. For small numbers of repeated measurements the relative standard deviation determined from the repeated measurements can itself have a large uncertainty (the variance of the variance) given by $s_{\text {rel }}=1 /$ sqrt [2(n-1)] where $n$ is the number of measurements. For example, the relative standard deviation of the observed standard deviation of 15 measurements is about $19 \%$.

\section{Measurement Results Summary}

The following three tables, Tables VIII-X, summarize all of the analyses of the recorded data. Each table summarizes a single detector. The samples are the plutonium samples listed previously in Table II. Most, but not all of the samples were measured on all three detectors. Fewer measurements were performed on Coaxial detector \#2 as it was not set up until the third day of the exercise. 
Table VIII. Data summary for planar detector

Planar

Sample:

Ratio:

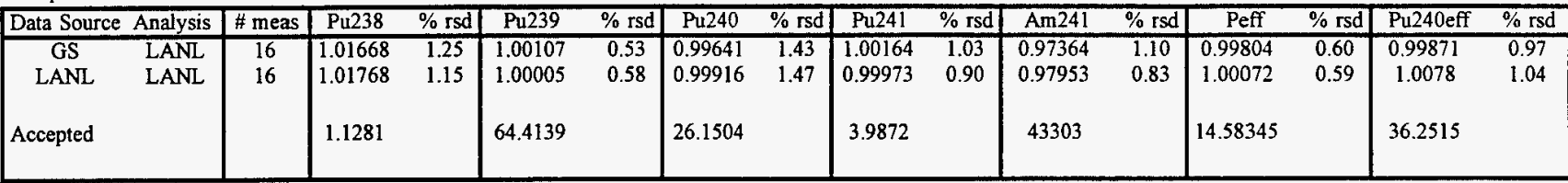

Sample:

Ratio:

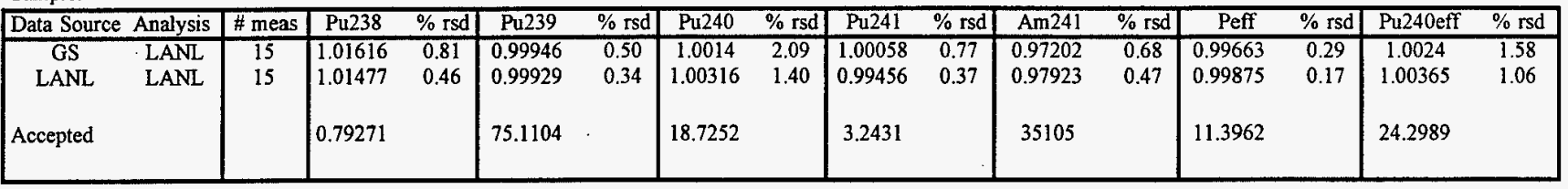

Sample: $\quad$ Ratio:

\begin{tabular}{|c|c|c|c|c|c|c|c|c|c|c|c|c|c|c|c|c|}
\hline Data Source & Analysis & \# meas & Pu238 & $\%$ rsd & Pu239 & $\%$ rsd & Pu240 & $\%$ rsd & Pu241 & $\%$ rsd & Am241 & \% rsd & Peff & $\%$ rsd & Pu240eff & $\%$ rsd \\
\hline GS & GS & 4 & 1.0242 & 3.70 & 1.0296 & 0.53 & 0.8452 & 3.98 & 1.0987 & 3.10 & 1.0828 & 6.14 & & & & \\
\hline GS & LANL & 4 & 0.98843 & 1.56 & 0.99773 & 0.20 & 1.01374 & 1.17 & 0.99497 & 0.37 & 0.97888 & 0.54 & 0.99741 & 0.08 & 1.0129 & 1.10 \\
\hline LANL & LANL & 4 & 1.00375 & 3.31 & 0.99726 & 0.14 & 1.01648 & 0.83 & 0.99454 & 0.30 & 0.98555 & 0.37 & 1.00073 & 0.25 & 1.0157 & 0.79 \\
\hline Accepted & & & 0.0646 & & 84.7192 & & 14.2588 & & 0.5981 & & 6446 & & 3.767558 & & 15.0253 & \\
\hline
\end{tabular}

Sample: $\quad$ Ratio:

\begin{tabular}{|c|c|c|c|c|c|c|c|c|c|c|c|c|c|c|c|c|}
\hline Data Source & Analysis & \# meas & Pu238 & $\%$ rsd & Pu239 & $\%$ rsd & Pu240 & $\%$ rsd & Pu24l & \% rsd & Am241 & $\%$ rsd & Peff & $\%$ rsd & Pu240eff & $\%$ rsd \\
\hline GS & GS & 15 & 1.0836 & 12.15 & 1.004 & 0.37 & $\overline{0.9374}$ & 5.78 & 1.0285 & 3.43 & 0.9692 & 15.09 & & & & \\
\hline GS & LANL & 15 & 1.0772 & 8.77 & 0.99968 & 0.16 & 1.0047 & 2.32 & 0.99502 & 0.47 & 0.98432 & 1.01 & 1.00101 & 0.33 & 1.00497 & 2.28 \\
\hline LANL & LANL & 15 & $\begin{array}{l}1.04112 \\
0.0107\end{array}$ & 6.17 & $\begin{array}{r}0.9996 \\
93.560\end{array}$ & 0.12 & $\begin{array}{r}1.00601 \\
6.314\end{array}$ & 1.80 & $\begin{array}{c}0.99342 \\
0.1296\end{array}$ & 0.25 & $\begin{array}{c}0.99769 \\
1962\end{array}$ & 0.85 & $\begin{array}{l}1.00151 \\
2.54007\end{array}$ & 0.24 & $\begin{array}{l}1.00611 \\
6.4074\end{array}$ & 1.77 \\
\hline
\end{tabular}

Sample: $\quad$ Ratio:

\begin{tabular}{|c|c|c|c|c|c|c|c|c|c|c|c|c|c|c|c|c|}
\hline Data Source & Analysis & \# meas & Pu238 & $\%$ rsd & Pu239 & $\%$ rsd & Pu240 & $\%$ rsd & Pu241 & $\%$ rsd & Am241 & $\%$ rsd & Peff & $\%$ rsd & Pu240eff & $\%$ rsd \\
\hline GS & GS & 5 & 0.834 & 11.73 & 7.0096 & 0.57 & 0.8526 & 10.30 & 1.1111 & 10.02 & 1.0697 & 9.52 & & & & \\
\hline GS & LANL & 5 & 0.71955 & 14.83 & 1.00242 & 0.20 & 0.96255 & 3.32 & 0.95583 & 0.77 & 0.99578 & 1.75 & 0.9873 & 0.34 & 0.96155 & 3.24 \\
\hline LANL & LANL & 4 & 0.73496 & 7.20 & 1.00105 & 0.23 & 0.98428 & 3.76 & 0.99124 & 0.74 & 0.99985 & 1.46 & 0.99079 & 0.27 & 0.98304 & 3.68 \\
\hline Accepted & & & 0.0133 & & 93.8479 & & 5.9529 & & 0.1524 & & 3266 & & 2.6854 & & 6.0427 & \\
\hline
\end{tabular}

Sample: $\quad$ Ratio:

\begin{tabular}{|c|c|c|c|c|c|c|c|c|c|c|c|c|c|c|c|c|}
\hline Data Source & Analysis & \# meas & Pu238 & $\%$ rsd & Pu239 & $\%$ rsd & Pu240 & $\%$ rsd & Pu241 & $\%$ rsd & Am241 & $\%$ rsd & Peff & $\%$ rsd & Pu240eff & $\%$ rsd \\
\hline GS & GS & 15 & 0.9988 & 5.57 & 1.0123 & 0.20 & 0.8124 & 4.01 & 1.0794 & 1.04 & 1.0101 & 11.22 & & & & \\
\hline GS & LANL & 15 & 0.9992 & 4.28 & 1.0004 & 0.09 & 0.9936 & 1.42 & 1.0011 & 0.31 & 1.0110 & 0.82 & 0.9999 & 0.22 & 0.9937 & 1.38 \\
\hline LANL & LANL & 15 & 0.99333 & 3.26 & 1.00037 & 0.07 & 0.99412 & 1.09 & 0.99867 & 0.30 & 1.02471 & 0.61 & 1.00052 & 0.15 & 0.99423 & 1.06 \\
\hline Accepted & & & 0.0135 & & 93.8749 & & 5.8638 & & 0.1818 & & 1170 & & 2.442242 & & 6.0085 & \\
\hline
\end{tabular}

Sample: $\quad$ Ratio:

\begin{tabular}{|c|c|c|c|c|c|c|c|c|c|c|c|c|c|c|c|c|}
\hline Data Source & Analysis & \# meas & Pu238 & \% rsd & $\mathrm{Pu} 239$ & $\%$ rsd & Pu240 & $\%$ rsd & Pu241 & $\%$ rsd & Am241 & \% rsd & Peff & $\%$ rsd & Pu240eff & $\%$ rsd \\
\hline GS & GS & 13 & 1.0052 & 5.13 & 1.0149 & 0.44 & 0.8616 & 4.85 & 1.0873 & 4.52 & 1.005 & 9.30 & & & & \\
\hline GS & LANL & 13 & 0.98854 & 4.15 & 1.00107 & 0.14 & 0.9896 & 1.37 & 0.99199 & 0.56 & 0.99436 & 0.82 & 0.99697 & 0.25 & 0.98979 & 1.34 \\
\hline LANL & LANL & 12 & 0.98447 & 2.36 & 1.00056 & 0.11 & 0.99474 & 1.11 & 0.99039 & 0.44 & 0.99735 & 0.74 & 0.99807 & 0.18 & 0.99478 & 1.09 \\
\hline Accepted & & & 0.0243 & & 90.552 & & 9.0101 & & 0.309 & & 4450 & & 3.041432 & & 9.247 & \\
\hline
\end{tabular}

\begin{tabular}{|c|c|c|c|c|c|c|c|c|c|c|c|c|c|c|c|c|}
\hline Sample: & & & & & & & & Ratio: & & & & & & & & \\
\hline Data Source & Analysis & \# meas & Pu238 & $\%$ rsd & Pu239 & $\%$ rsd & Pu240 & $\%$ rsd & Pu241 & $\%$ rsd & Am241 & $\%$ rsd & Peff & $\%$ rsd & Pu240eff & \% rsd \\
\hline $\begin{array}{l}\text { GS } \\
\text { GS }\end{array}$ & $\begin{array}{c}\text { GS } \\
\text { LANL }\end{array}$ & $\begin{array}{l}15 \\
15\end{array}$ & $\begin{array}{c}0.9657 \\
1.00949\end{array}$ & $\begin{array}{l}7.03 \\
3.39\end{array}$ & $\begin{array}{c}1.0058 \\
1.00079\end{array}$ & $\begin{array}{l}0.16 \\
0.04\end{array}$ & $\begin{array}{r}0.8469 \\
0.97857\end{array}$ & $\begin{array}{l}5.22 \\
1.05\end{array}$ & $\begin{array}{c}1.0691 \\
1.00494\end{array}$ & $\begin{array}{l}.28 \\
0.27\end{array}$ & $\begin{array}{c}0.9986 \\
1.02367\end{array}$ & $\begin{array}{l}19.46 \\
1.07\end{array}$ & 0.9991 & 0.11 & 0.97887 & 1.03 \\
\hline LANL & LANL & 15 & 1.0153 & 3.33 & 1.00068 & 0.04 & 0.98162 & 1.04 & 1.0149 & 0.27 & 1.049 & 0.99 & 1.00026 & 0.10 & 0.98181 & 1.03 \\
\hline Accepted & & & 0.0059 & & 96.3504 & & 3.5609 & & 0.0645 & & 631 & & 2.218442 & & 3.6065 & \\
\hline
\end{tabular}


Coaxial \#1 (hand held) Detecto 'Data

Table IX. Data summary for Coaxial Detector \#1

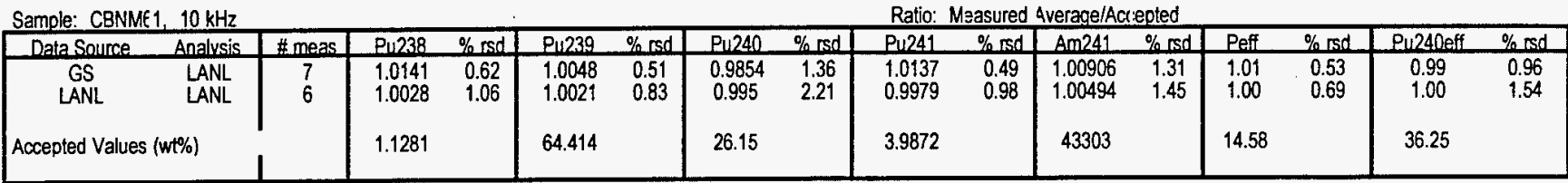

Sample: CBNME4, $10 \mathrm{xHz}$

Ratio: Measured Average/Acreoted

\begin{tabular}{|c|c|c|c|c|c|c|c|c|c|c|c|c|c|c|c|c|}
\hline Data Source & Analusis & \#meas & Pu238 & $\%$ rsd & Pu239 & $\% \operatorname{csd}$ & $P_{14240}$ & $\%$ rsd & Pu241 & $\% \operatorname{ssd}$ & Am241 & $\% \mathrm{rd}$ & Peff & $\% \operatorname{rsd}$ & Pu240eff & \% rsd \\
\hline $\begin{array}{c}\text { GS } \\
\text { GS } \\
\text { LANL }\end{array}$ & $\begin{array}{l}\text { GS } \\
\text { LANL } \\
\text { LANL }\end{array}$ & $\begin{array}{l}15 \\
15 \\
15\end{array}$ & $\begin{array}{l}1.0215 \\
0.9905 \\
0.9852\end{array}$ & $\begin{array}{l}6.15 \\
1.82 \\
2.83\end{array}$ & $\begin{array}{l}1.0054 \\
1.001 \\
1.0012\end{array}$ & $\begin{array}{l}1.31 \\
0.22 \\
0.13\end{array}$ & $\begin{array}{l}0.9922 \\
0.9939 \\
0.9929\end{array}$ & $\begin{array}{c}7.67 \\
1.3 \\
0.77\end{array}$ & $\begin{array}{l}1.0255 \\
1.0039 \\
0.9971\end{array}$ & $\begin{array}{l}4.89 \\
0.4 \\
0.3\end{array}$ & $\begin{array}{c}0.9737 \\
0.98518 \\
0.99616\end{array}$ & $\begin{array}{l}7.58 \\
0.69 \\
0.72\end{array}$ & $\begin{array}{c}0.995 \\
0.9964\end{array}$ & $\begin{array}{l}0.3 \\
0.4\end{array}$ & $\begin{array}{l}0.9941 \\
0.9931\end{array}$ & $\begin{array}{l}1.24 \\
0.74\end{array}$ \\
\hline Accepted Value & & & 0.0646 & & 84.719 & & 14.259 & & 0.5981 & & 6446 & & 3.7676 & & 15.025 & \\
\hline
\end{tabular}

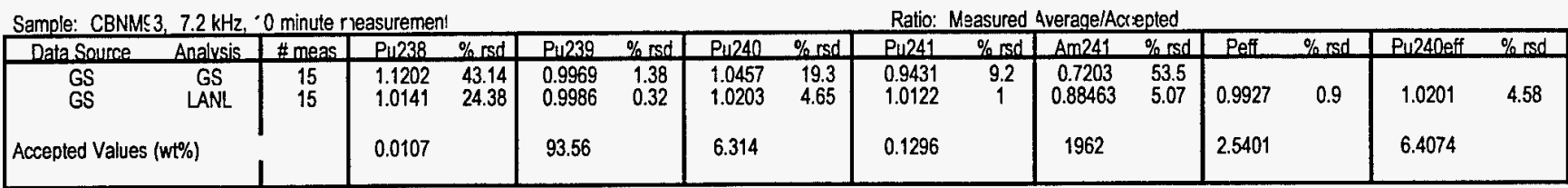

Sample: CBNMS3, $25 \mathrm{kHz}$

Ratio: Measured Average/Accepted

\begin{tabular}{|c|c|c|c|c|c|c|c|c|c|c|c|c|c|c|c|c|}
\hline Data Source & Analusis & \#meas & Pu238 & $\%$ rsd & Pu239 & $\%$ rsd & Pu240 & $\% \mathrm{rsd}$ & Pu241 & $\% \mathrm{ssd}$ & Am241 & $\%$ nd & Peff & $\% \sqrt{s d}$ & Pu240eff & $\%$ ssd \\
\hline $\begin{array}{c}\text { GS } \\
\text { GS } \\
\text { LANL }\end{array}$ & $\begin{array}{l}\text { GS } \\
\text { LANL } \\
\text { LANL }\end{array}$ & $\begin{array}{c}8 \\
8 \\
15\end{array}$ & $\begin{array}{l}1.027 \\
1.011 \\
1.059\end{array}$ & $\begin{array}{l}13.81 \\
8.33 \\
5.55\end{array}$ & $\begin{array}{l}0.998 \\
0.999 \\
0.999\end{array}$ & $\begin{array}{l}0.6 \\
0.19 \\
0.12\end{array}$ & $\begin{array}{l}1.036 \\
1.009 \\
1.009\end{array}$ & $\begin{array}{l}8.43 \\
2.86 \\
1.83\end{array}$ & $\begin{array}{l}0.9120 \\
1.0118 \\
0.9995\end{array}$ & $\begin{array}{l}5.5 \\
0.3 \\
0.3\end{array}$ & $\begin{array}{l}0.906 \\
0.897 \\
0.976\end{array}$ & $\begin{array}{l}16.5 \\
2.24 \\
1.48\end{array}$ & $\begin{array}{l}0.9923 \\
1.0003\end{array}$ & $\begin{array}{l}0.3 \\
0.2\end{array}$ & $\begin{array}{l}1.009 \\
1.009\end{array}$ & $\begin{array}{l}2.81 \\
1.8\end{array}$ \\
\hline Accepted Value & & & 0.0107 & & 93.506 & & 6.314 & & 0.1296 & & 1962 & & 2.5401 & & 6.407 & \\
\hline
\end{tabular}

Sample: METSFH, $4 \mathrm{kHz}$

\begin{tabular}{|c|c|c|c|c|c|c|c|c|c|c|c|c|c|c|c|c|}
\hline Data Source & Analysis & \#meas & P11238 & $\%$ rsd & Pu1239 & $\%$ rsd & Pu240 & $\% \mathrm{rd}$ & Pu241 & $\%$ rsd & Am241 & $\% \mathrm{rd}$ & Peff & $\%$ rsd & Pi240eff & $\%$ asd \\
\hline $\begin{array}{c}\text { GS } \\
\text { GS } \\
\text { LANL }\end{array}$ & $\begin{array}{l}\text { GS } \\
\text { LANL } \\
\text { LANL }\end{array}$ & $\begin{array}{l}15 \\
15 \\
15\end{array}$ & $\begin{array}{l}0.7659 \\
0.7447 \\
0.7343\end{array}$ & $\begin{array}{l}21.25 \\
13.93 \\
13.36\end{array}$ & $\begin{array}{l}0.998 \\
0.999 \\
0.9991\end{array}$ & $\begin{array}{l}0.69 \\
0.21 \\
0.11\end{array}$ & $\begin{array}{l}1.0391 \\
1.0157 \\
1.0145\end{array}$ & $\begin{array}{l}10.3 \\
3.28 \\
1.69\end{array}$ & $\begin{array}{l}0.9206 \\
\text { t.0072 } \\
0.9983\end{array}$ & $\begin{array}{l}6.2 \\
0.4 \\
0.3\end{array}$ & $\begin{array}{l}0.9122 \\
0.92783 \\
0.96577\end{array}$ & $\begin{array}{l}15.1 \\
1.94 \\
0.7\end{array}$ & $\begin{array}{l}0.9847 \\
0.9894\end{array}$ & $\begin{array}{l}0.5 \\
0.3\end{array}$ & $\begin{array}{c}1.014 \\
1.0125\end{array}$ & $\begin{array}{l}3.28 \\
1.67\end{array}$ \\
\hline Accepted Value & & & 0.0133 & & 93.848 & & 5.9529 & & 0.1524 & & 3266 & & 2.6854 & & 6.0427 & \\
\hline
\end{tabular}

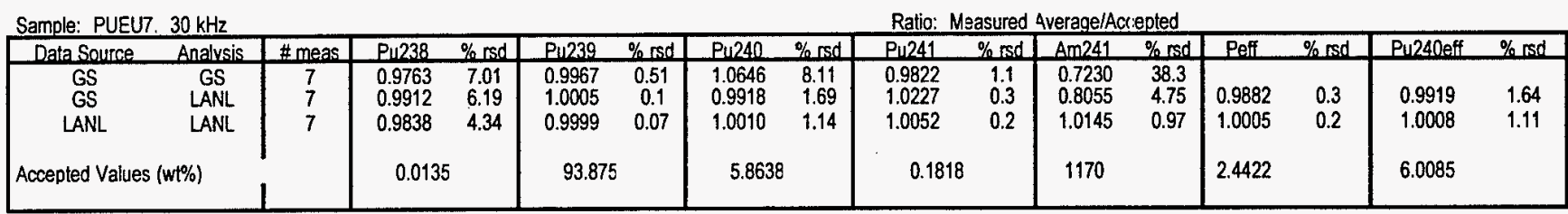

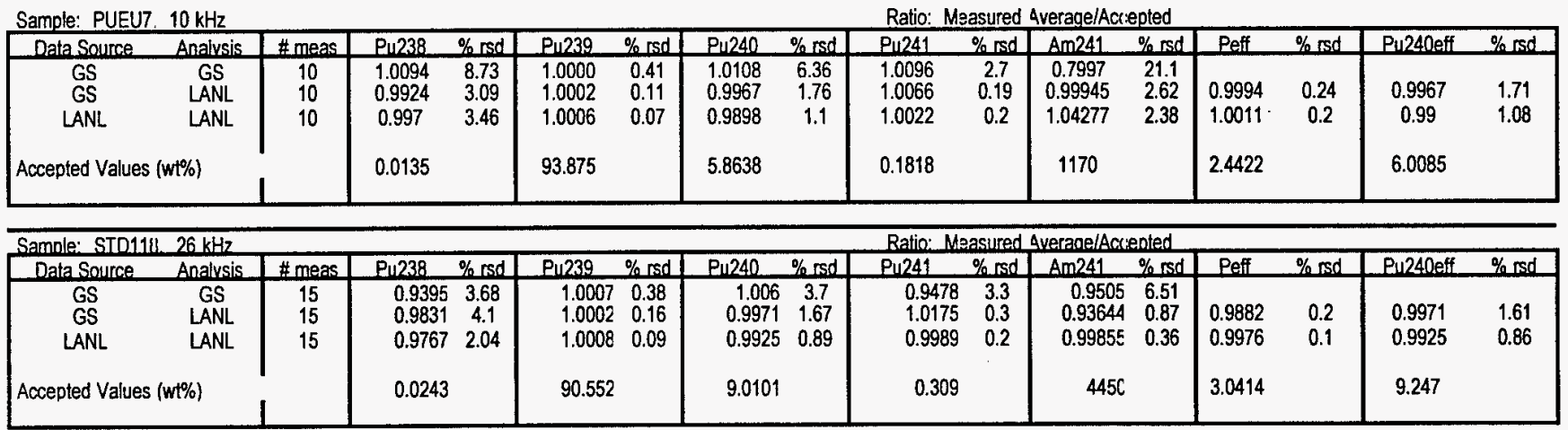

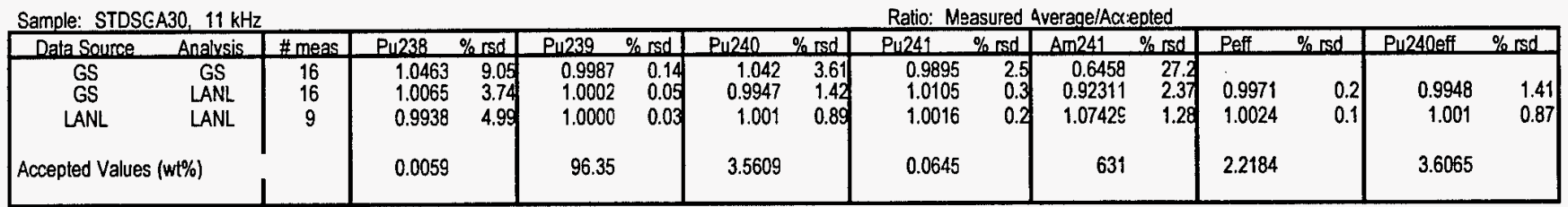


Coaxial \#2 (JSPEC) Datector

Table X. Data summary for Coaxial Detector \#2

Sample: C3NM61, $: 0 \mathrm{kHz}$

Ratio: Measured Average Accepted

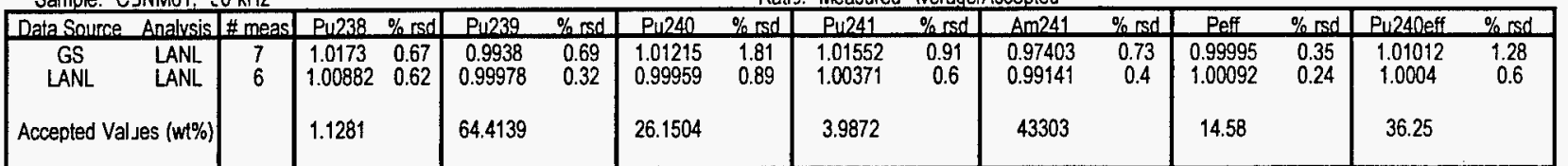

Sample: $\mathrm{C} 3 \mathrm{NM} 70,: 0 \mathrm{kHz}$

Ratix: Measured 4verage, Accepted

\begin{tabular}{|c|c|c|c|c|c|c|c|c|c|c|c|c|c|c|}
\hline Data Source & \#meas & $P(1238$ & Pu239 & $\% \mathrm{rsd}$ & Pu240 & $\%$ rso & Pu241 & $\%$ rsd & Am241 & $\% \mathrm{ss}$ & Peff & $\%$ rs & P11240eff & $\% \times s$ \\
\hline $\begin{array}{l}\text { GS } \\
\text { LANL }\end{array}$ & $\begin{array}{c}16 \\
3\end{array}$ & $\begin{array}{cc}1.01031 & 1.1 \\
1.0052 & 0.22\end{array}$ & $\begin{array}{l}0.99642 \\
0.99712\end{array}$ & $\begin{array}{l}0.67 \\
0.29\end{array}$ & $\begin{array}{l}1.01235 \\
1.01119\end{array}$ & $\begin{array}{l}2.8 \\
1.2\end{array}$ & $\begin{array}{l}1.00917 \\
1.00085\end{array}$ & $\begin{array}{l}0.81 \\
0.33\end{array}$ & $\begin{array}{r}0.98528 \\
0.9873\end{array}$ & $\begin{array}{l}0.96 \\
0.93\end{array}$ & $\begin{array}{l}0.99996 \\
0.99852\end{array}$ & $\begin{array}{l}0.45 \\
0.24\end{array}$ & $\begin{array}{l}1.01036 \\
1.00905\end{array}$ & $\begin{array}{l}2.11 \\
0.92\end{array}$ \\
\hline Accepted Val Jes (wt\%) & & 0.79271 & 75.1104 & & 18.75252 & & 3.2431 & & 35105 & & 11.3962 & & 0.242989 & \\
\hline
\end{tabular}

Sample: C3NM84, $10 \mathrm{kHz}$

Ratio: Measured Averace. Accepted

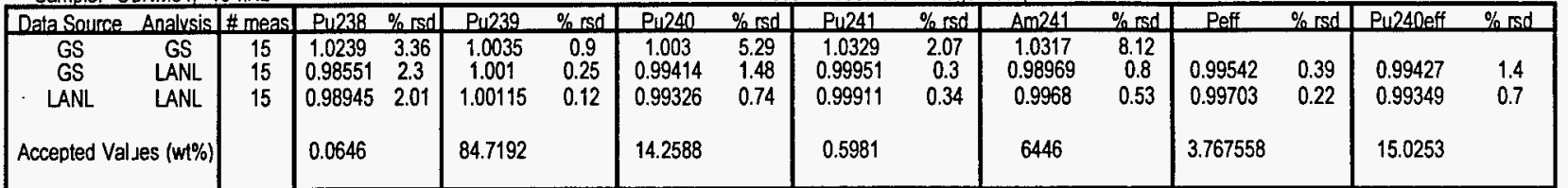

Sample: C3NM93, $50 \mathrm{kHz}$

Ratio: Measured Average Accepted

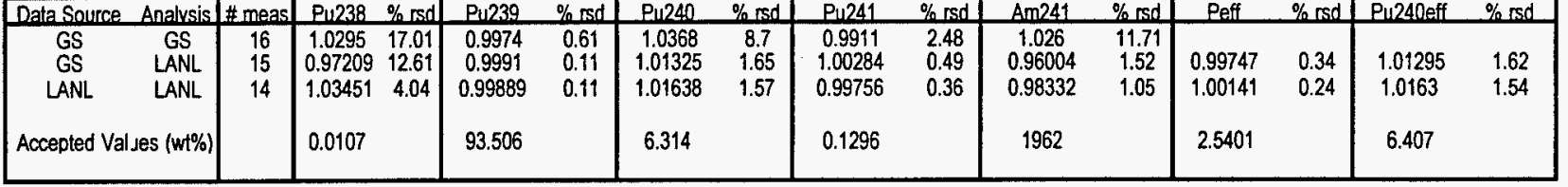

Sample: (BNM93 1) kHz

Ratio: Measured Averace Accepted

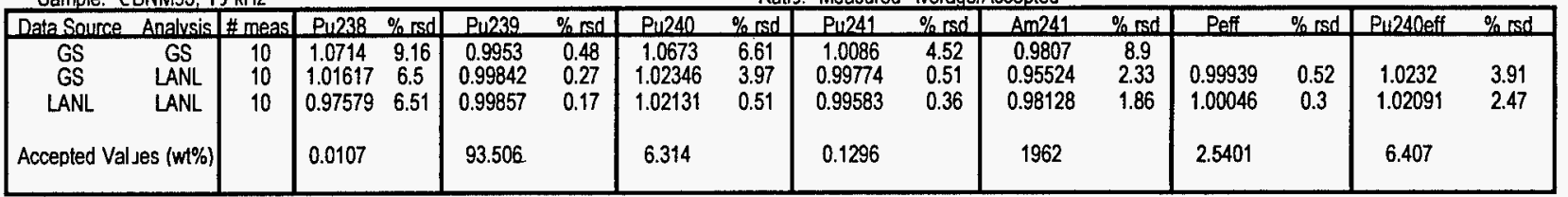

Sample: METSPH, $\cdot 6 \mathrm{kHz}$

Ratis: Measured 4verage, Accepted

\begin{tabular}{|c|c|c|c|c|c|c|c|c|c|c|c|c|c|c|}
\hline Data Source Analvsis & \#meas & Pu238 & $P \cup 239$ & $\%$ ssd & $\mathrm{P}, 240$ & $\%$ asd & 201241 & $\%$ rsd & Am241 & $\%$ asd & Peff & $\%$ rsd & Pu240eff & $\% \mathrm{rdd}$ \\
\hline $\begin{array}{cc}\text { GS } & \text { GS } \\
\text { GS } & \text { LANL }\end{array}$ & $\begin{array}{l}16 \\
16 \\
9\end{array}$ & $\begin{array}{ll}0.7192 & 16.56 \\
0.73548 & 51.14 \\
0.74837 & 9.23\end{array}$ & $\begin{array}{l}0.9986 \\
0.99852 \\
0.99827\end{array}$ & $\begin{array}{l}0.55 \\
0.17 \\
0.12\end{array}$ & $\begin{array}{c}1.029 \\
1.02399 \\
1.02798\end{array}$ & $\begin{array}{l}8.36 \\
2.67 \\
1.87\end{array}$ & $\begin{array}{r}0.9561 \\
0.99819 \\
0.99564\end{array}$ & $\begin{array}{l}6.79 \\
0.38 \\
0.32\end{array}$ & $\begin{array}{l}0.9801 \\
0.95998 \\
0.97181\end{array}$ & $\begin{array}{l}13.88 \\
1.48 \\
0.68\end{array}$ & $\begin{array}{c}0.9898 \\
0.99225\end{array}$ & $\begin{array}{l}0.47 \\
0.38\end{array}$ & $\begin{array}{l}1.02217 \\
1.02617\end{array}$ & $\begin{array}{l}2.66 \\
185\end{array}$ \\
\hline Accepted Val Jes (wt\%) & & 0.0133 & 93.8479 & & $\begin{array}{l}1.02170 \\
5.9529\end{array}$ & & 0.1524 & & 3266 & & 2.6854 & & 6.0427 & \\
\hline
\end{tabular}

Sample: ST JSGA30, $10 \mathrm{kHz}$

Ratis: Measured Average, Accepted

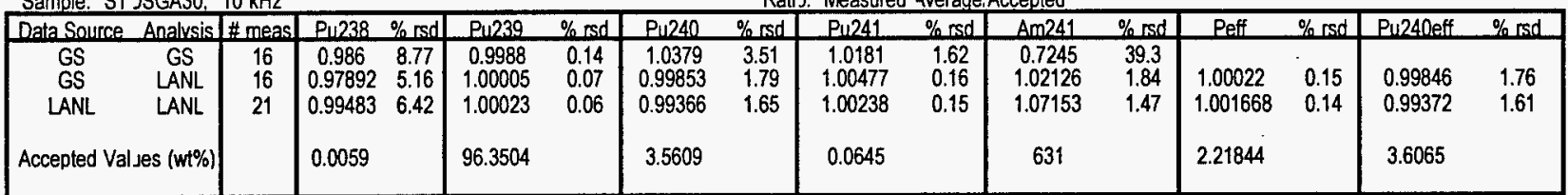

In the figures below we plot some of the data from the tables just presented. The graphical presentations emphasize the quantities most important for Materials Control and Accountability (MC\&A) measurements on bulk materials, ${ }^{240} \mathrm{Pu}$ and the effective specific power $P_{\text {eff }}(\mathrm{W} / \mathrm{gPu})$. The Greenstar analysis did not calculate $\mathrm{P}_{\text {eff }}$ thus this quantity is not presented for the Green Star data source.

There are significant trends in the precision of the results from the three combinations of measurements/analysis. The Green Star data-Green Star analysis shows the lowest precision, resulting from the different goals for its development and the analysis approach taken. In comparing the Green Star data-LANL analysis with the LANL data-LANL analysis we observe the effect of lower throughput of the Green Star data acquisition electronics relative to the LANL electronics. In nearly every case, the Green Star data-LANL analysis has poorer precision than the LANL data-LANL analysis. 


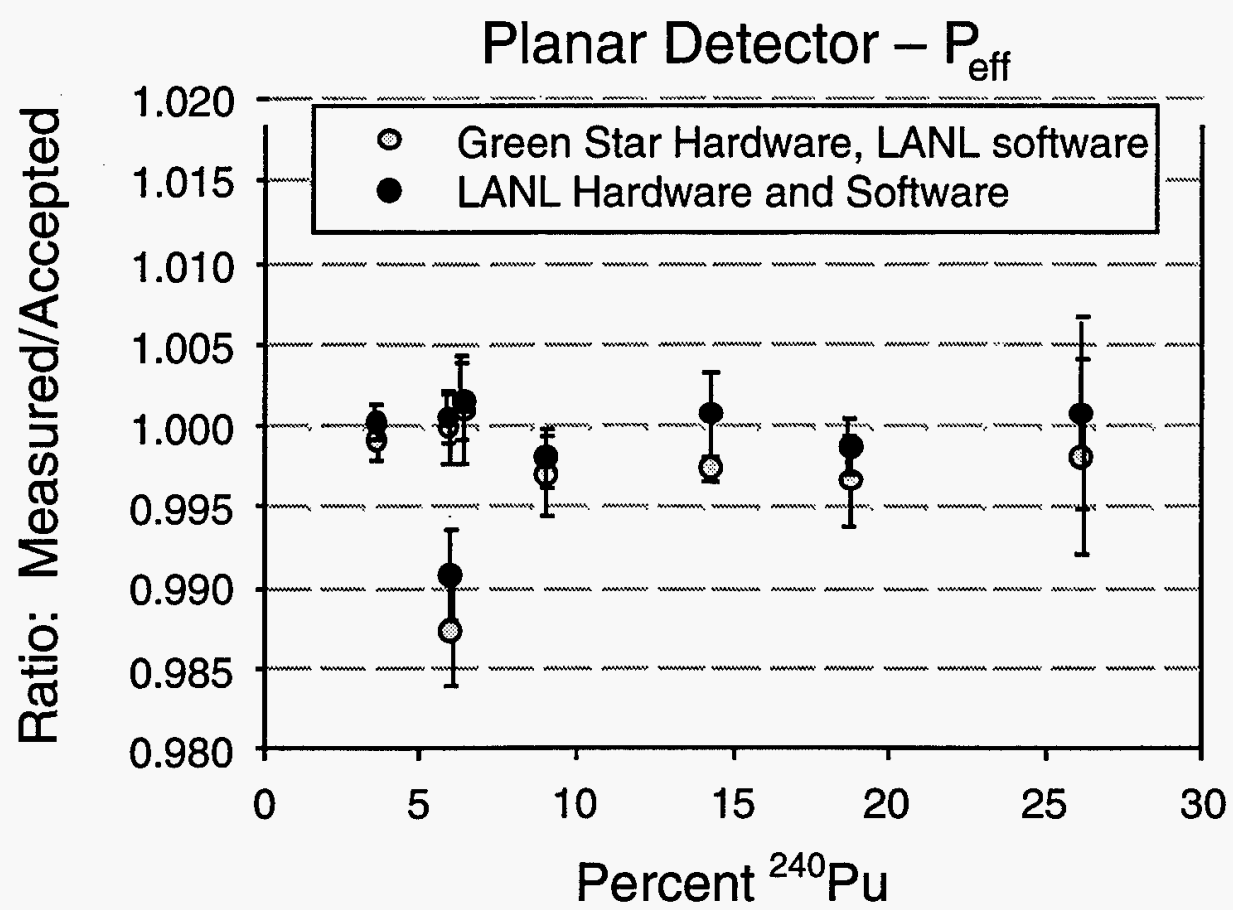

Fig. 7. Ratio of measured/accepted values of $P_{\text {eff }}$ for LANL PC/FRAM analysis of LANL and Green Star data from the planar detector. The low points are from the METSPH sample, believed to have an incorrect ${ }^{238} \mathrm{Pu}$ value (see Table II).

In Figs. 7-15 the error bars that are plotted are the standard deviation of a single measurement from the \%rsd values in the tables presented above.

The LANL analysis of both LANL and Green Star data from the planar detector (Fig. 7,8) is consistent with the historic performance of the PC/FRAM code. The Green Star analysis shows a significant $15 \%$ low bias on the planar detector. This bias does not appear for the Green Star coaxial detector data. A possible explanation arises from the steeper relative efficiency curve for the planar detector relative to the coaxial detector. The Green Star software was developed for the more efficient coaxial detector and may have some difficulty with the planar detector's relative efficiency curve. It is also believed that the high bias for ${ }^{241} \mathrm{Pu}$ on the planar detector arises in the same fashion and the two effects are interrelated.

There appears to be small, but consistent difference between the LANL analyses of the LANL and Green Star data. $P_{\text {eff }}$ for the LANL analysis of LANL data is consistently higher than for the LANL analysis of the Green Star data. The same effect is present in the ${ }^{240} \mathrm{Pu}$ data. While the differences are insignificant in the practical sense, it would be interesting to investigate whether they may be caused by the lack of a pile-up rejector on the Green Star system.

We also noted, in the summary tables, Tables VIII-X, differences between the ${ }^{241} \mathrm{Am}$ values for the Green Star-LANL and the LANL-LANL analyses. The differences are such that the LANL-LANL values are consistently higher than the Green StarLANL values for most of the data from the two coaxial detectors. The planar data does not show this effect. One hypothesis for this effect is that it may arise from summing in the larger coaxial detector and that the differences are affected by the lack of a pile up rejector on the Green Star electronics. 


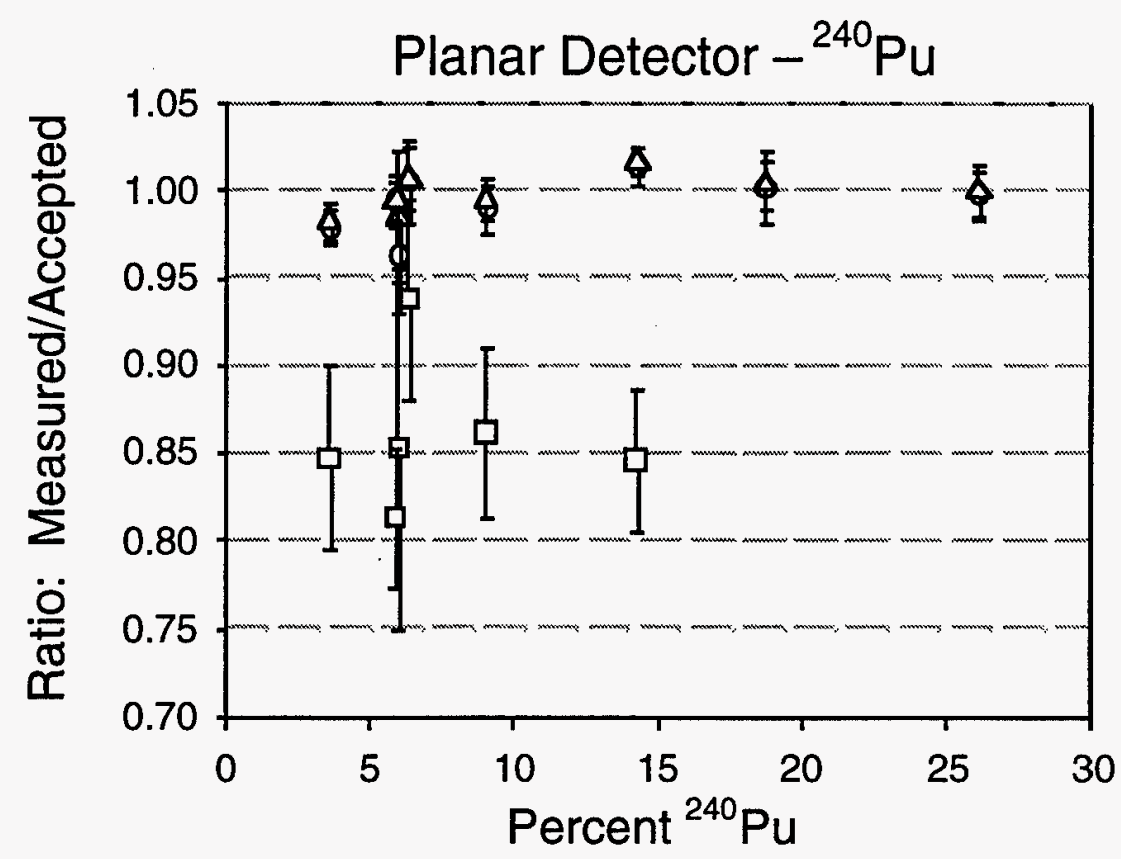

\section{Green Star Hardware and Software \\ - Green Star Hardware, LANL Software \\ $\triangle \quad$ LANL Hardware and Software}

Fig. 8. Ratio of measured/accepted values of ${ }^{240} \mathrm{Pu}$ for LANL PC/FRAM analysis of LANL and Green Star data and the Green Star analysis of Green Star data. All data from the planar detector.

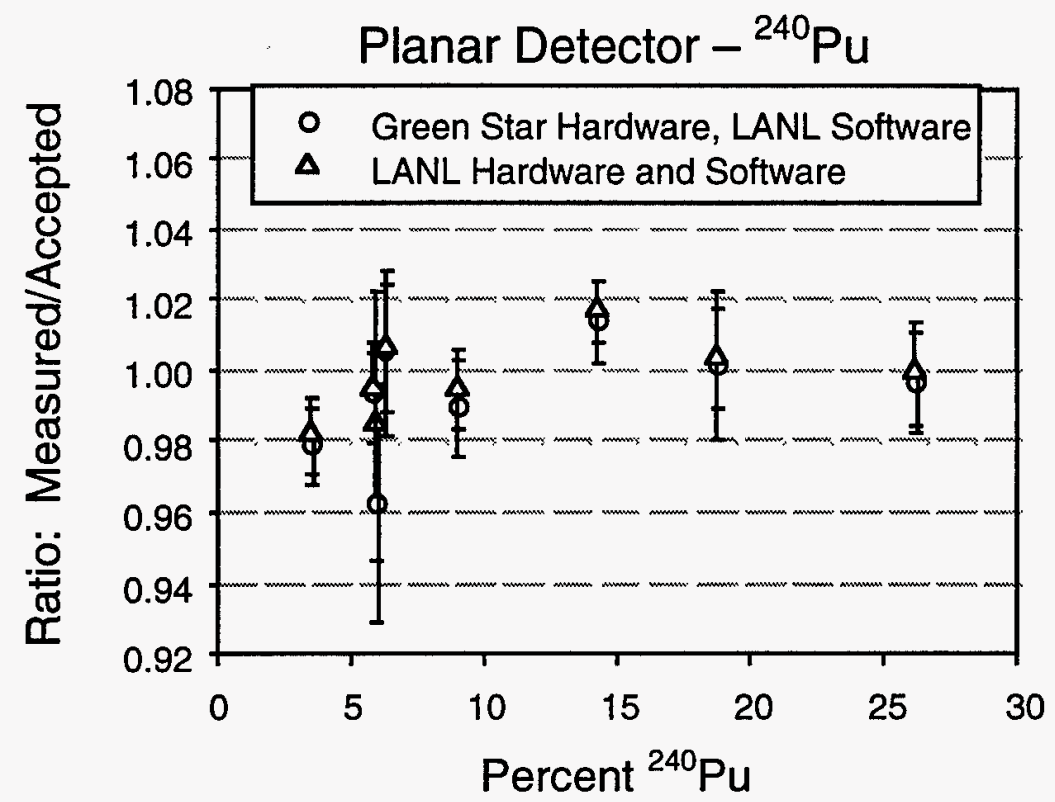

Fig. 9. Expanded view of the LANL analysis in Fig. 8. 


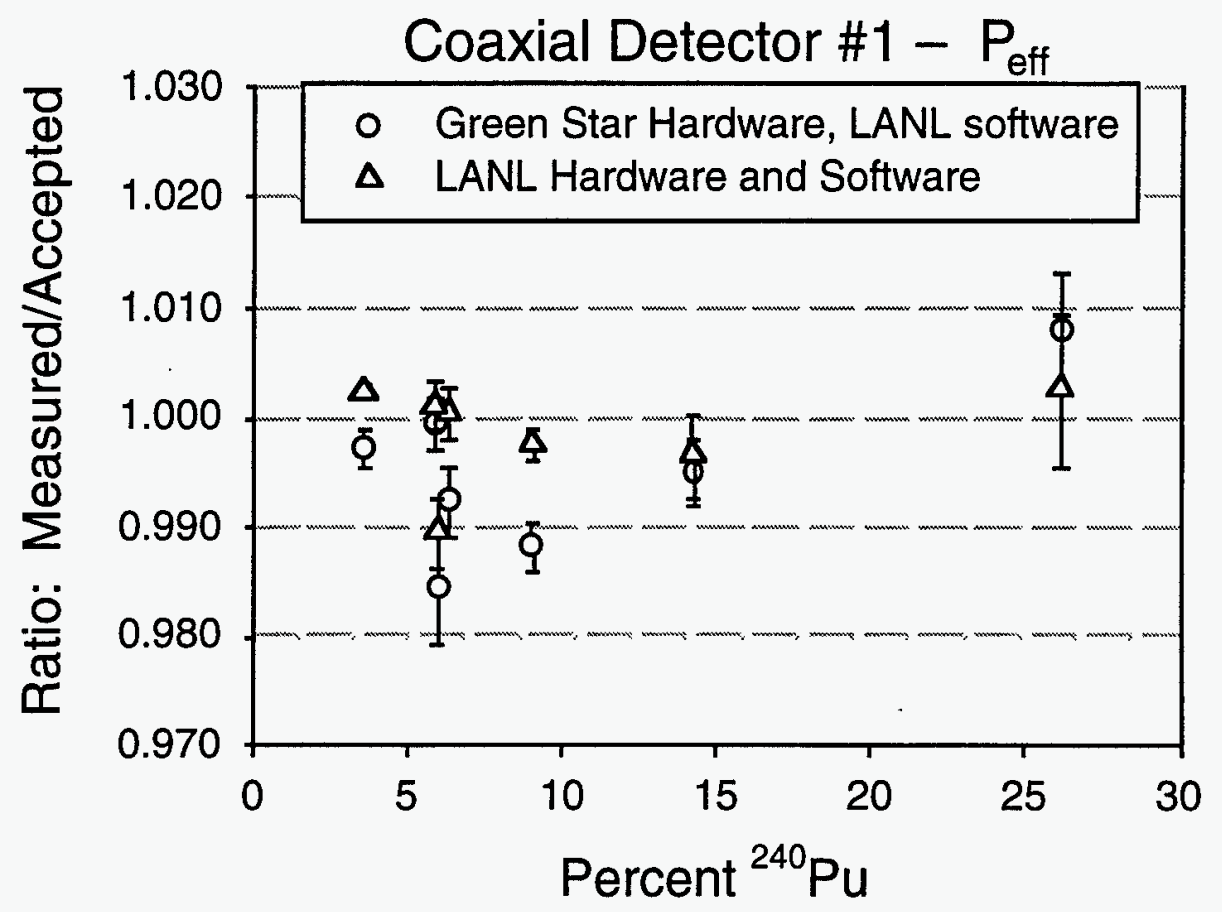

Fig. 10. Ratio of measured/accepted values of $P_{\text {eff }}$ for LANL PC/FRAM analysis of LANL and Green Star data from coaxial detector \#1.

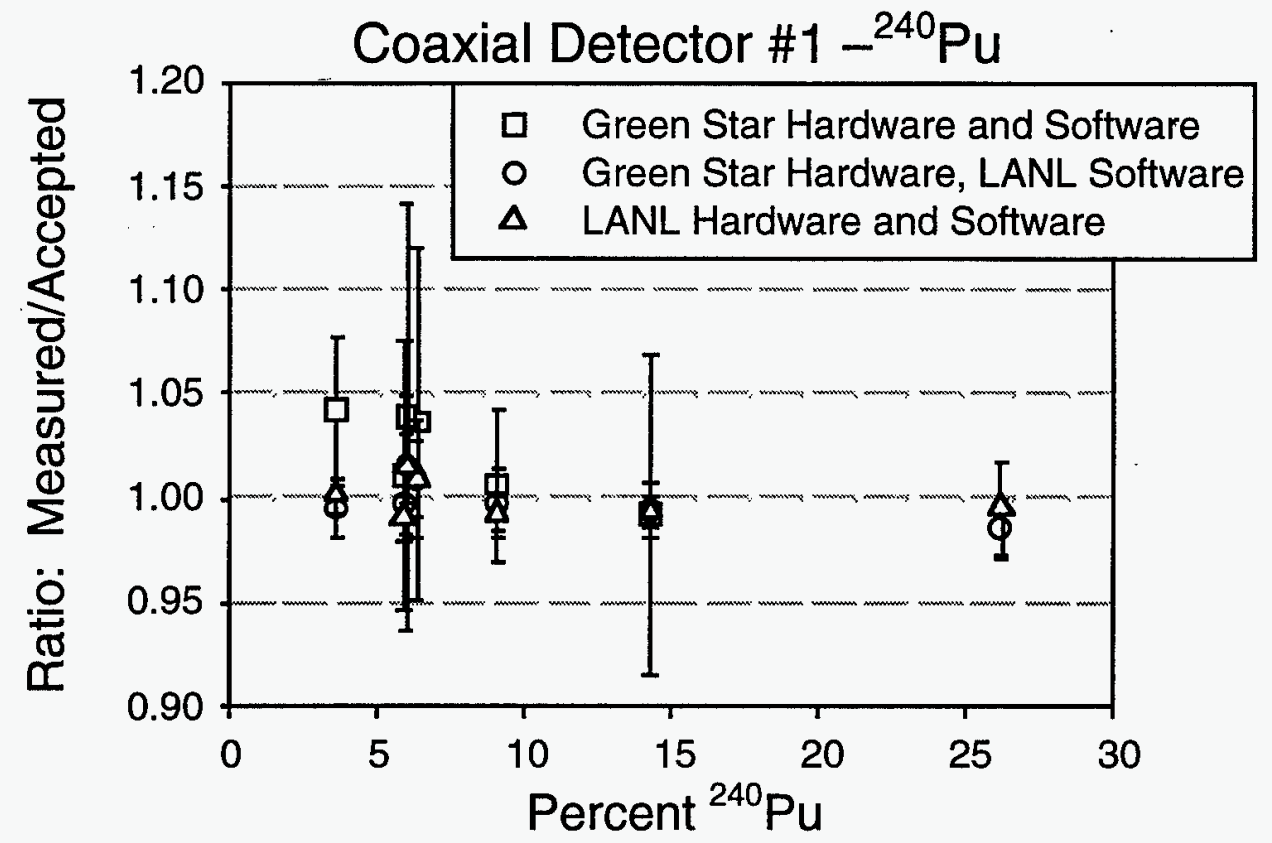

Fig. 11. All analyses for ${ }^{240} \mathrm{Pu}$ from Coaxial Detector \#1. 


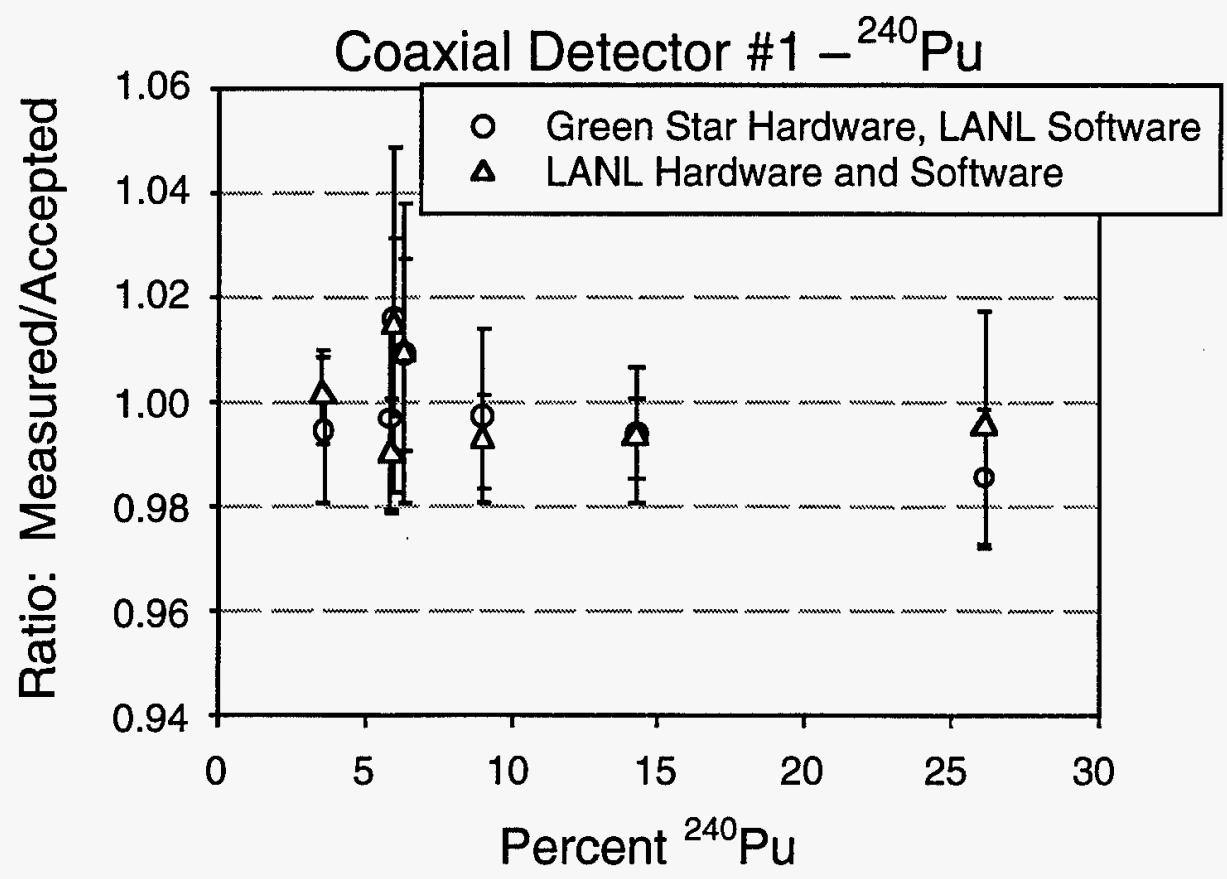

Fig. 12. Expanded view of ${ }^{240} \mathrm{Pu}$ analyses from Coaxial Detector \#1.

The data for Coaxial Detector \#1 continue to show the expected ${ }^{240} \mathrm{Pu}$ accuracy $(<1 \%)$ for the PC/FRAM analyses independent of the data acquisition hardware. For Peff, Fig. 10, the LANL hardware gave better accuracy averaged over all samples as well as better precision compared to the Green Star hardware.

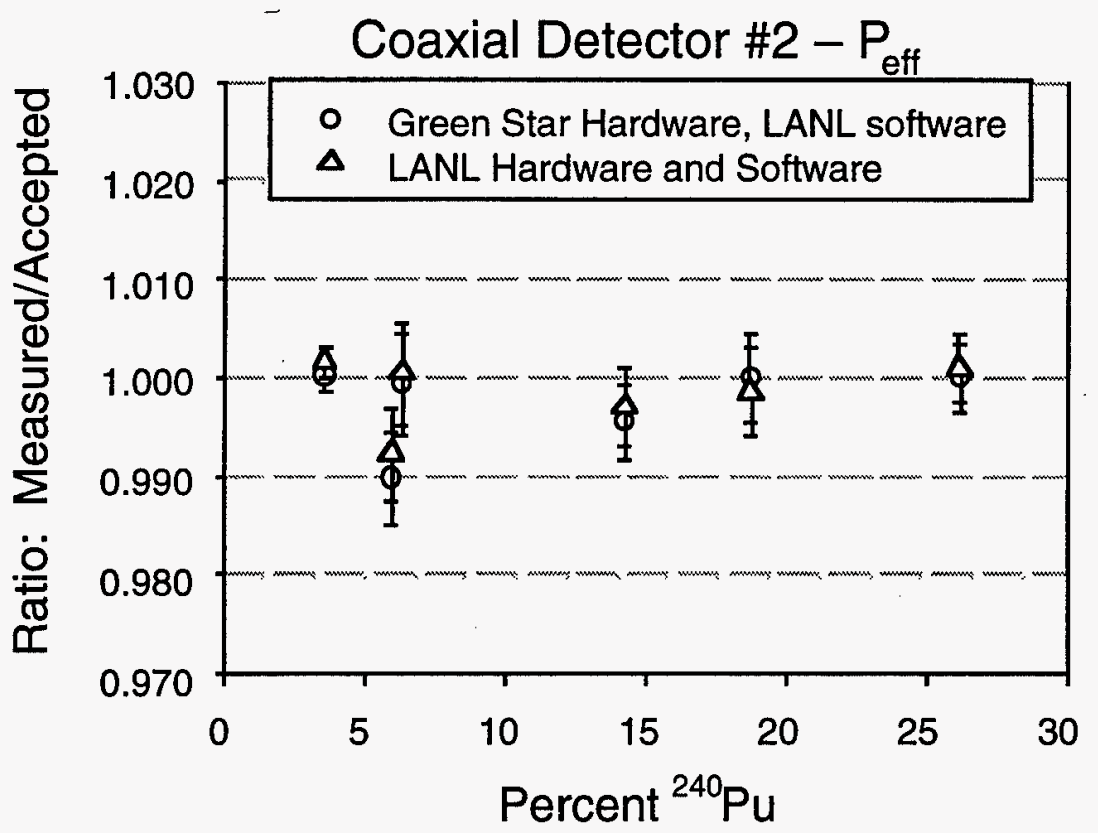

Fig. 13. Ratio of measured/accepted values of $P_{\text {eff }}$ for LANL PC/FRAM analysis of LANL and Green Star data from Coaxial Detector \#2. 


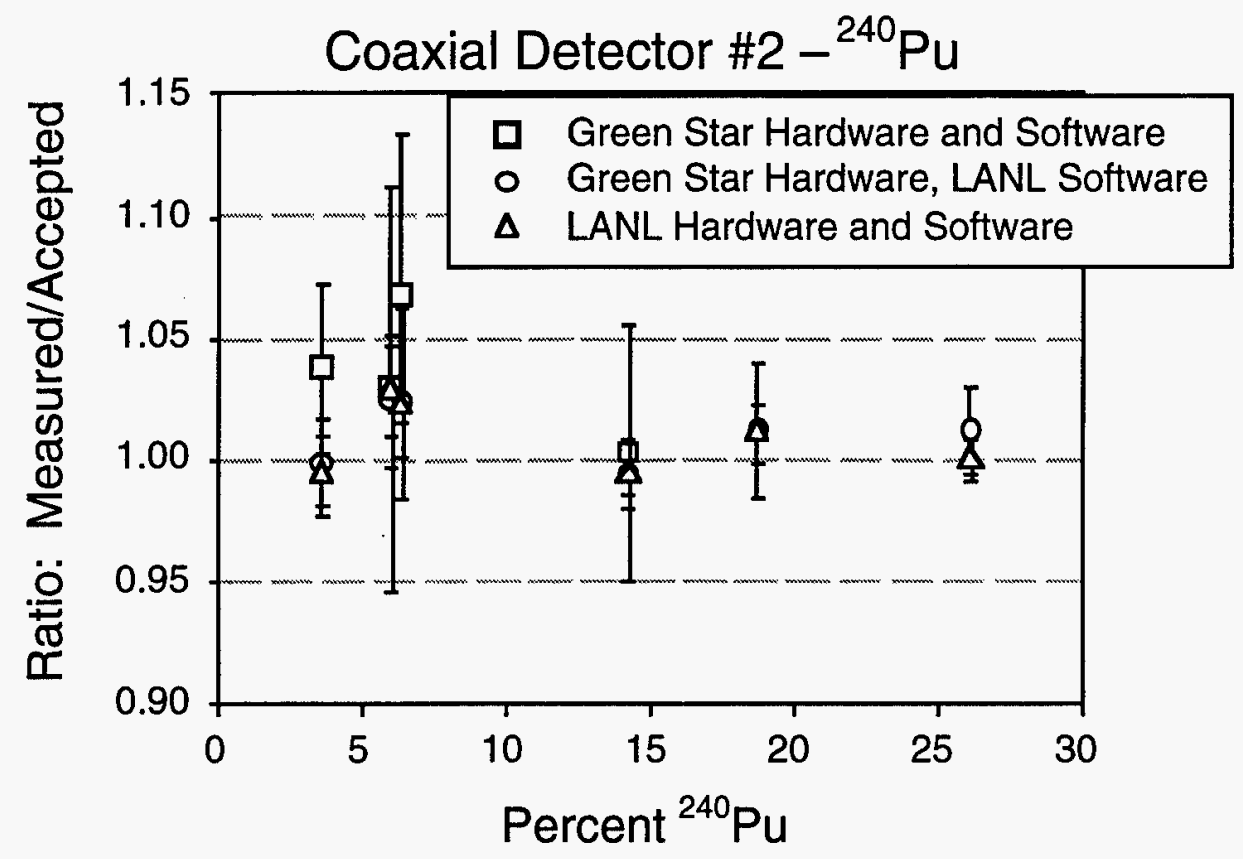

Fig. 14. All analyses for ${ }^{240} \mathrm{Pu}$ from Coaxial Detector \#2.

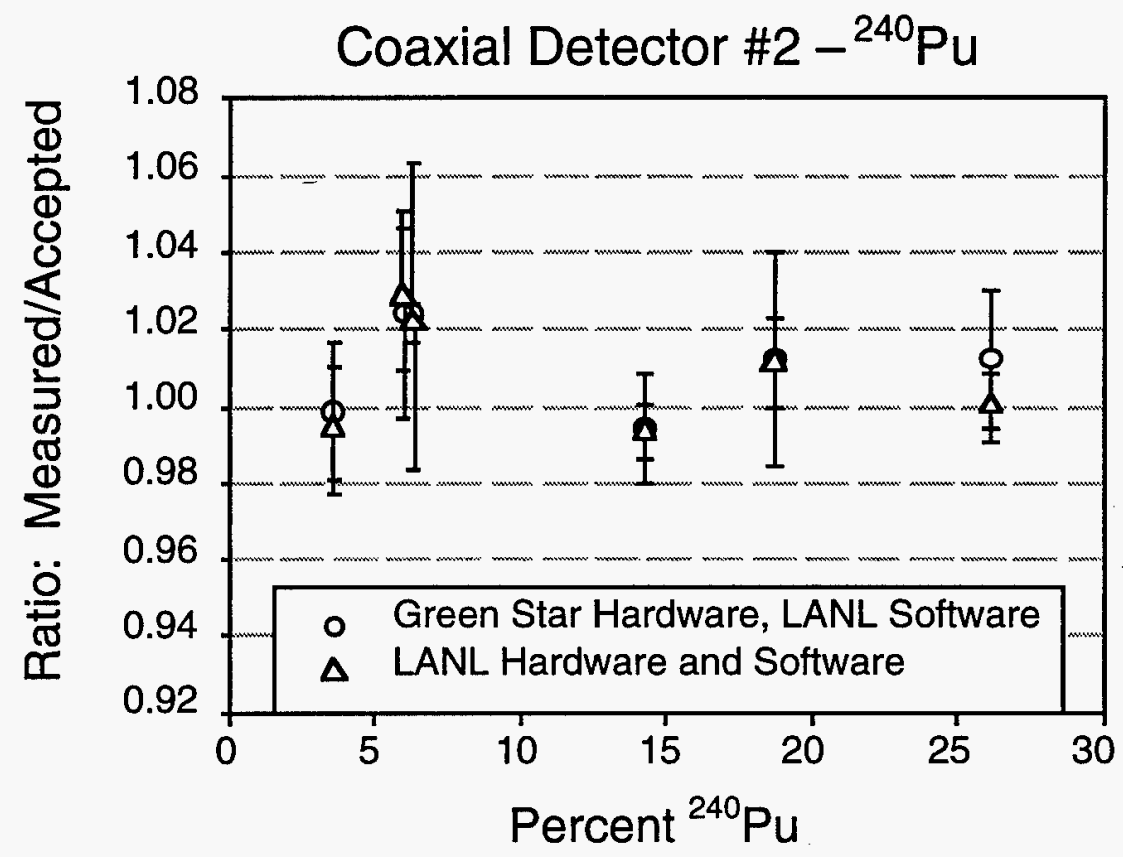

Fig. 15. Expanded view of ${ }^{240} \mathrm{Pu}$ analyses from Coaxial Detector $\# 2$. 
Table XI below presents for each detector the unweighted average over all samples of the bias (Measured Average/Accepted) for each isotope. The \%RSD is that of the distribution of the individual sample (Measured Average/Accepted) values about the average for all samples. As such it represents a standard deviation of the bias and has contributions from the statistical uncertainty of the individual average values as well contributions from the true sample-sample biases.

The ${ }^{238} \mathrm{Pu}$ value for METSPH has not been used in the averages in Table XI.

\begin{tabular}{|c|c|c|c|c|c|c|c|c|c|c|}
\hline \multicolumn{3}{|c|}{ Planar Detector } & \multicolumn{8}{|c|}{ Unweighted Average Bias for All Samples } \\
\hline Data/Analysis & Pu238 & $\%$ RSD & Pu239 & \%RSD & Pu240 & $\% \mathrm{RSD}$ & Pu241 & $\%$ RSD & $\overline{\mathrm{Am} 24}$ & \%RSD \\
\hline GS/GS & $\overline{1.016}$ & 4.3 & 1.013 & 0.9 & 0.859 & 4.9 & 1.079 & 2.7 & 1.023 & 4.3 \\
\hline GS/LANL & 1.011 & 3.1 & 1.000 & 0.14 & 0.994 & 1.5 & 0.992 & 1.5 & 0.988 & 1.3 \\
\hline LANL/LANL & 1.009 & 2.0 & 1.000 & 0.12 & 0.998 & 1.1 & 0.996 & 0.82 & 0.998 & 2.2 \\
\hline \multicolumn{3}{|c|}{ Coax\#1 Detector } & \multicolumn{8}{|c|}{ Unweighted Average Bias for All Samples } \\
\hline Data/Analysis & $\mathrm{Pu} 238$ & $\%$ RSD & $\overline{\text { Pu239 }}$ & $\%$ RSD & $\overline{P u 240}$ & \%RSD & $\overline{\text { Pu241 }}$ & \%RSD & Am24 & \%RSD \\
\hline GS/GS & 1.020 & 5.6 & 0.999 & 0.29 & 1.030 & 2.3 & 0.966 & 4.3 & 0.829 & 14.8 \\
\hline GS/LANL & 1.000 & 1.2 & 1.000 & 0.18 & 1.000 & 1.2 & 1.012 & 0.57 & 0.930 & 6.9 \\
\hline LANL/LANL & 1.000 & 2.8 & 1.000 & 0.10 & 0.999 & 0.86 & 1.000 & 0.27 & 1.009 & 3.5 \\
\hline \multicolumn{3}{|c|}{ Coax\#2 Detector } & \multicolumn{8}{|c|}{ Unweighted Average Bias for All Samples } \\
\hline$\overline{\text { Data/Analysis }}$ & Pu238 & $\%$ RSD & $\overline{\text { Pu239 }}$ & \%RSD & Pu240 & $\%$ RSD & Pu241 & $\%$ RSD & Am24 & \%RSD \\
\hline$\overline{\mathrm{GS} / \mathrm{GS}}$ & 1.028 & 3.4 & 0.999 & $\overline{0.30}$ & 1.035 & $\overline{2.2}$ & 1.001 & 2.9 & 0.949 & 13.4 \\
\hline GS/LANL & 0.997 & 2.0 & -0.998 & 0.24 & 1.011 & 1.1 & 1.004 & 0.65 & 0.978 & 2.4 \\
\hline LANL/LANL & 1.001 & 2.0 & 0.999 & 0.13 & 1.009 & 1.4 & 0.999 & 0.32 & 0.998 & 3.4 \\
\hline
\end{tabular}

\section{Conclusions}

Overall, the Green Star spectrometer performed very well, especially considering that its electronics and software were designed for low counting rate applications, that of identification, and not for the higher performance requirements of the exacting quantification required for MC\&A applications.

Throughput was significantly lower for the Green Star spectrometer compared to the LANL NIM bin and DSPEC systems. Green Star suggested a "best" counting rate for their system of $10 \mathrm{kHz}$ while LANL suggests $30 \mathrm{kHz}$ for coaxial systems at $2 \mu \mathrm{s}$ and $40 \mathrm{kHz}$ for planar detectors at their usual time constant of $1 \mu \mathrm{s}$. (LANL actually used $2 \mu \mathrm{s}$ for its planar detector data acquisition to match the time constant used by Green Star.) Higher counting rate capability than $10 \mathrm{kHz}$ will be required for the larger samples to be encountered in dismantlement applications. The effect of the lower throughput was seen to be poorer precision for the Green Star data compared with the LANL data when analyzed with the same software.

In contrast to the poorer throughput, the resolution of the Green Star system was better than that of the LANL systems in many instances.

It is possible that the lack of a pile up rejector in the tested Green Star systems caused some problems in the analysis of ${ }^{241} \mathrm{Am}$, in particular. More study of the data is needed to confirm this. In any case, a pile up rejector should be used for all MC\&A measurements. Future Green Star systems should be tested with their optional pile up rejector. 
In the comparisons of the accuracy and precision of the analysis for ${ }^{240} \mathrm{Pu}$ and $\mathrm{P}_{\text {eff }}$ with the PC/FRAM code, the Green Star acquisitions and the LANL acquisitions gave comparable results, with the exception of the precision discussed above. This is a significant conclusion because it means that the Green Star system could acquire data for the PC/FRAM application with little degredation in performance compared to the US systems tested.

\section{Recommendations}

Green Star should immediately proceed with the development of their SBS-60 spectrometer with a faster successive approximations $\mathrm{ADC}$. The faster $\mathrm{ADC}$ is required for best precision for MC\&A measurements on the large items expected from dismantlement.

Green Star should target their developments toward improving the counting rate capability of their system for use in MC\&A applications.

All Green Star spectrometers for use in MC\&A applications should incorporate a pile up rejector.

The SBS-60 spectrometer with pile up rejector should be tested at Los Alamos, much in the same way that these tests were conducted. These tests should be conducted as soon as the new system is operational but only after the system is thoroughly tested and debugged in Russia.

Successful testing of the SBS-60 would allow it to be the data acquisition device for isotopic analysis measurements with PC/FRAM software for use in material disposition applications. 


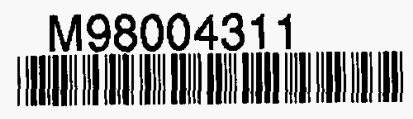

Report Number (14) $L A-4 R--97-5026$

Publ. Date (11) $1997 / 2$
Sponsor Code (18)
UC Category (19) $4 C-700, X F$
UC , DOE/ER 